ESAIM: COCV 27 (2021) 21

https://doi.org/10.1051/cocv/2021016
ESAIM: Control, Optimisation and Calculus of Variations

www.esaim-cocv.org

\title{
GLOBAL GRADIENT ESTIMATES FOR NONLINEAR PARABOLIC OPERATORS
}

\author{
Serena Dipierro ${ }^{1, *}, \mathrm{Zu}_{\mathrm{GaO}}^{2}$ And Enrico VAldinoci ${ }^{1}$
}

\begin{abstract}
We consider a parabolic equation driven by a nonlinear diffusive operator and we obtain a gradient estimate in the domain where the equation takes place. This estimate depends on the structural constants of the equation, on the geometry of the ambient space and on the initial and boundary data. As a byproduct, one easily obtains a universal interior estimate, not depending on the parabolic data. The setting taken into account includes sourcing terms and general diffusion coefficients. The results are new, to the best of our knowledge, even in the Euclidean setting, though we treat here also the case of a complete Riemannian manifold.
\end{abstract}

Mathematics Subject Classification. 35B09, 35B50, 35K05, 35R01.

Received September 6, 2020. Accepted February 4, 2021.

\section{INTRODUCTION}

The heat equation was introduced almost two centuries ago by Joseph Fourier [3]. In spite of its classical flavor, the investigation of the main properties of the solution is still an active field of research, and several important gradient estimates have been obtained in modern literature. Also, given its importance in geometric evolution problems, some of these results have been framed into the framework of Riemannian manifolds. Among the several results on this topic, we recall the following universal bound for compact manifolds:

Theorem 1.1 (Thm. 1.1 in [15]). Let $\mathcal{M}$ be a compact Riemannian manifold with $\operatorname{Ric}(\mathcal{M}) \geq-k$, for some $k \geq 0$. Let $u=u(x, t)$ be a positive solution of $u_{t}=\Delta u$ in $\mathcal{M} \times(0,+\infty)$. Assume that $u \leq M$ for some $M>0$.

Then, for each $(x, t) \in \mathcal{M} \times(0,+\infty)$,

$$
\frac{t|\nabla u(x, t)|^{2}}{u^{2}(x, t)} \leq(1+2 k t) \ln \frac{M}{u(x, t)} .
$$

This type of result is certainly striking and also somewhat surprising, since typically parabolic estimates aim at controlling positive solutions at a given time by values at a later time, in view of the diffusive character of the equation (see e.g. the classical Harnack Inequality on page 89 of [12]), while Richard Hamilton's estimate in (1.1) is a pointwise estimate in space-time.

Keywords and phrases: Parabolic equations on Riemannian manifolds, maximum principle, global gradient estimates.

1 Department of Mathematics and Statistics, University of Western Australia, 35 Stirling Highway, 6009 Crawley, Australia.

2 Department of Mathematics, School of Science, Wuhan University of Technology, 122 Luoshi Road, 430070 Hubei, Wuhan, P.R. China.

* Corresponding author: serena.dipierro@uwa.edu.au 
As a matter of fact, an estimate of this type cannot hold in non-compact manifolds, as the simple case of the fundamental solution in $\mathbb{R}^{n}$ shows: namely, taking $u(x, t):=\frac{1}{(4 \pi t)^{\frac{n}{2}}} \exp \left(-\frac{|x|^{2}}{4 t}\right)$, one sees that

$$
\frac{t|\nabla u|^{2}}{u^{2}}=\frac{|x|^{2}}{4 t}
$$

which does not permit a global bound as in (1.1).

With respect to this, several gradient estimates have been obtained in non-compact manifolds by considering "interior estimates" in space and time. More specifically, if $x_{0} \in \mathcal{M}$ and $R>0$, one denotes the geodesic ball of radius $R$ centered at $x_{0}$ by $B\left(x_{0}, R\right)$. Also, given $t_{0} \in \mathbb{R}$ and $T>0$, we let

$$
Q_{R, T}:=B\left(x_{0}, R\right) \times\left[t_{0}-T, t_{0}\right] .
$$

In this setting, a fruitful topic of investigation consists in obtaining local gradient estimates in $Q_{R / 2, T / 2}$, see especially the work [18] by Peter Li and Shing-Tung Yau in which the maximum principle and suitable cut-off functions have been used to obtain a parabolic Harnack inequality on complete Riemannian manifolds. In this setting, we recall also a celebrated result by Philippe Souplet and Qi S. Zhang:

Theorem 1.2 (Thm. 1.1 in [24]). Let $\mathcal{N}$ be a complete Riemannian manifold with $\operatorname{Ric}(\mathcal{M}) \geq-k$, for some $k \geq 0$. Let $u=u(x, t)$ be a positive solution of $u_{t}=\Delta u$ in $Q_{R, T}$. Assume that $u \leq M$ for some $M>0$.

Then, for each $(x, t) \in Q_{R / 2, T / 2}$,

$$
\frac{|\nabla u(x, t)|}{u(x, t)} \leq C\left(\frac{1}{R}+\frac{1}{\sqrt{T}}+\sqrt{k}\right)\left(1+\ln \frac{M}{u(x, t)}\right)
$$

for a suitable positive dimensional constant $C$.

This result has been extended by Li Ma, Lin Zhao and Xianfa Song [22] to the case of nonlinear equations, obtaining the following structural result:

Theorem 1.3 (Thm. 7 in [22]). Let $\mathcal{M}$ be a complete Riemannian manifold of dimension $n$ with $\operatorname{Ric}(\mathcal{M}) \geq-k$, for some $k \geq 0$. Let $u=u(x, t)$ be a positive solution of $u_{t}=\Delta(F(u))$ in $Q_{R, T}$, with $F \in C^{2}(0,+\infty)$. Assume that $u \leq M$ for some $M>0$ and that

$$
F^{\prime}(s) \in(0, K] \text { for every } s \in(0, M]
$$

Let also $G:(0,+\infty) \rightarrow \mathbb{R}$ be such that $G^{\prime}(s)=F^{\prime}(s) / s$ for all $s \in(0, M]$ and suppose that

$$
\begin{array}{ll} 
& 1-\frac{\sqrt{n}\left|F^{\prime \prime}(s)\right| s}{F^{\prime}(s)} \geq \kappa>0, \\
& \xi-G(s) \geq \eta>0 \\
\text { and } \quad 2 F^{\prime}(s)-\frac{\sqrt{n}\left|F^{\prime \prime}(s)\right| s}{F^{\prime}(s)}(\xi-G(s))>0
\end{array}
$$

for every $s \in(0, M]$, for suitable constants $\kappa, \eta, \xi$.

Then, there exists $C>0$, depending only on $n, K, \kappa$ and $\eta$ such that, for each $(x, t) \in Q_{R / 2, T / 2}$,

$$
\frac{|\nabla G(u(x, t))|}{\xi-G(u(x, t))} \leq C\left(\frac{1}{R}+\frac{1}{\sqrt{T}}+\sqrt{k}\right) .
$$


As detailed in Remark 8 in [22] (see also Appendix A here), Theorem 1.3 includes Theorem 1.2 as a special case, when $F(s)=s$. Moreover, while conditions (1.4) may look rather technical at a first glance, they are in fact sufficiently general to treat several important nonlinear models such as the porous medium equation $u_{t}=\Delta u^{p}$ (see e.g. [27], and also [5, 6, 13, 14] for the case of Riemannian manifolds) with

$$
p \in\left(1-\frac{1}{\sqrt{n}}, 1\right]
$$

In this case, Theorem 1.3 entails the following statement:

Corollary 1.4 (Cor. 9 in [22]). Let $\mathcal{M}$ be a complete Riemannian manifold of dimension 2 and 3 , with $\operatorname{Ric}(\mathcal{N}) \geq$ $-k$, for some $k \geq 0$. Let $u=u(x, t)$ be a positive solution of $u_{t}=\Delta u^{p}$ in $Q_{R, T}$, with $p$ as in (1.6). Assume that $u \leq M$ for some $M>0$.

Then, there exists $C>0$, depending only on $n$ and $p$ such that, for each $(x, t) \in Q_{R / 2, T / 2}$,

$$
\frac{|\nabla u(x, t)|}{u(x, t)} \leq C\left(\frac{1}{R}+\frac{M^{\frac{1-p}{2}}}{\sqrt{T}}+\sqrt{k}\right) .
$$

For the sake of precision, we observe that, strictly speaking, in the original formulation given in [22], Corollary 9 in [22] is not a direct consequence of Theorem 7 in [22], since the proof of the corollary presented there does not rely merely on the statement of the theorem but rather on a skillful modification of its proof: nevertheless, it is possible to deduce the corollary directly from the results that we will present here, as we point out in Appendix A.

We recall that existence and uniqueness results for the porous medium equation with $p<1$ have been established in [16]. We also mention that universal pointwise estimates for porous medium equations have been obtained in [1]. In [19] the authors prove, together with other gradient estimates on manifolds, that one can derive from these universal pointwise estimates also gradient estimates in the case of fast diffusion.

See also $[2,4,7-10,17,20,21,23,25,28-32]$ for parabolic estimates related to the results presented so far. The goal of this article is to enhance Theorem 1.3 (and consequently Thm. 1.2) in several directions:

- First of all, we replace the nonlinear operator $\Delta(F(u))$ with the more general nonlinear diffusive term

$$
a(x, t, u) \Delta(F(u))
$$

Even when $F(s)=s$, this improvement is interesting since it corresponds to allowing a heat equation in which the diffusion coefficient of the substratum depends on space, time, and possibly also the temperature;

- Moreover, we allow a source term depending on space, time, on the solution itself, and possibly also on the gradient and the Hessian of the solution;

- In addition, instead of a local estimate, we obtain a global estimate in $Q_{R, T}$, depending on the parabolic data of the equation, which recovers the universal estimate in $Q_{R / 2, T / 2}$ as a byproduct.

To obtain our result, we will perform a number of rather involved and ad-hoc computations and exploit also the cut-off function method that was introduced in [10] to address global estimates. We also remark that, as far as we know, our results are new also in the case of nonlinear parabolic equations in the Euclidean space when $k=0$.

Our result relies on suitable structural assumptions, that can be seen as natural counterparts of those in (1.4), and, to state clearly the estimates obtained, we now introduce precisely the mathematical framework in which we work.

We consider the evolution equation

$$
u_{t}=a(x, t, u) \Delta(F(u))+H\left(x, t, u, \nabla u, D^{2} u\right)
$$


on a complete Riemannian manifold $\mathcal{M}$ of dimension $n$ and such that

$$
\operatorname{Ric}(\mathcal{M}) \geq-k
$$

for some $k \in \mathbb{R}$. In this notation, $u=u(x, t)$, where $x \in \mathcal{M}$ is the space variable and $t$ is the time variable. As customary, the notation " $\nabla$ " and " $\Delta$ " is reserved, respectively, for the gradient and the Laplacian in the space variable. We suppose that equation (1.8) is satisfied for every $(x, t) \in Q_{R, T} \subset \mathcal{M} \times(-\infty, \infty)$, where $Q_{R, T}$ was introduced in (1.2).

We take $a \in C^{1}\left(Q_{R, T} \times \mathbb{R}\right)$ with

$$
a(x, t, s) \in\left[a_{0}, a_{0}^{-1}\right]
$$

for all $(x, t, s) \in Q_{R, T} \times \mathbb{R}$, for some $a_{0} \in(0,1)$.

We suppose that the solution $u$ is smooth, positive and bounded, namely, that for every $(x, t) \in Q_{R, T}$ we have $u(x, t) \in(0, M]$, for some $M>0$.

We suppose that $F \in C^{2}(0,+\infty)$ with

$$
F^{\prime}(s)>0
$$

and

$$
1-\frac{\sqrt{n}\left|F^{\prime \prime}(s)\right| s}{F^{\prime}(s)} \geq \kappa>0
$$

for all $s \in(0, M]$, for a positive constant $\kappa$, and that $H \in C^{1}\left(Q_{R, T} \times \mathbb{R} \times \mathbb{R}^{n} \times \mathbb{R}^{n^{2}}\right)$. With respect to the variables of $H$, the "gradient-Hessian" coordinates in $\mathbb{R}^{n} \times \mathbb{R}^{n^{2}}$ will be denoted by

$$
(\omega, \Omega)=\left(\left(\omega_{i}\right)_{i \in\{1, \ldots, n\}},\left(\Omega_{i j}\right)_{i, j \in\{1, \ldots, n\}}\right) .
$$

We also take $s_{0} \in(0,+\infty)$ and define, for all $s \in(0, M]$,

$$
G(s):=\int_{s_{0}}^{s} \frac{F^{\prime}(h)}{h} d h
$$

and we assume that

$$
\xi-G(s) \geq \eta>0
$$

that

$$
\frac{F^{\prime}(s)}{\xi-G(s)} \leq \Gamma
$$

and that

$$
2 F^{\prime}(s)-\frac{\sqrt{n}\left|F^{\prime \prime}(s)\right| s}{F^{\prime}(s)}(\xi-G(s)) \geq 0
$$


for all $s \in(0, M]$, for a suitable ${ }^{1}$ constant $\xi$ and positive constants $\eta$ and $\Gamma$.

We introduce the structural constants

$$
\begin{aligned}
\mu_{1}:=\sup _{(x, t) \in Q_{R, T}}(k a(x, & t, u(x, t)) F^{\prime}(u(x, t))+\frac{H\left(x, t, u(x, t), \nabla u(x, t), D^{2} u(x, t)\right) F^{\prime \prime}(u(x, t))}{F^{\prime}(u(x, t))} \\
& +\partial_{u} H\left(x, t, u(x, t), \nabla u(x, t), D^{2} u(x, t)\right) \\
& -\frac{H\left(x, t, u(x, t), \nabla u(x, t), D^{2} u(x, t)\right)}{u(x, t)} \\
& \left.+\frac{H\left(x, t, u(x, t), \nabla u(x, t), D^{2} u(x, t)\right) F^{\prime}(u(x, t))}{(\xi-G(u(x, t))) u(x, t)}\right)_{+}
\end{aligned}
$$

and

$$
\gamma_{1}:=\sup _{(x, t) \in Q_{R, T}} \frac{F^{\prime}(u(x, t))\left|\nabla H\left(x, t, u(x, t), \nabla u(x, t), D^{2} u(x, t)\right)\right|}{u(x, t)} .
$$

Let also

$$
\mu_{2}:=\sup _{(x, t) \in Q_{R, T}}\left|\partial_{u} a(x, t, u(x, t))\right|\left|\operatorname{div}\left(F^{\prime}(u(x, t)) \nabla u(x, t)\right)\right|
$$

and

$$
\mu:=\mu_{1}+\mu_{2}
$$

We stress that $\mu_{2}=0$, and thus $\mu=\mu_{1}$, when $a$ depends only on $x$ and $t$ (but is independent of $u$ ).

We also consider the quantities

$$
\gamma_{2}:=\sup _{(x, t) \in Q_{R, T}} \frac{F^{\prime}(u(x, t))}{u(x, t)}|\nabla a(x, t, u(x, t))|\left|\operatorname{div}\left(F^{\prime}(u(x, t)) \nabla u(x, t)\right)\right|
$$

and

$$
\begin{aligned}
\gamma_{3}:=\sup _{(x, t) \in Q_{R, T}} \frac{F^{\prime}(u(x, t))}{u(x, t)}\left(\left|\nabla_{\omega} H\left(x, t, u(x, t), \nabla u(x, t), D^{2} u(x, t)\right)\right|\left|D^{2} u(x, t)\right|\right. \\
\left.\quad+\left|D_{\Omega} H\left(x, t, u(x, t), \nabla u(x, t), D^{2} u(x, t)\right)\right|\left|D^{3} u(x, t)\right|\right)
\end{aligned}
$$

and we set

$$
\gamma:=\gamma_{1}+\gamma_{2}+\gamma_{3}
$$

\footnotetext{
${ }^{1}$ It is interesting to remark that conditions (1.3) and (1.4) are stronger than conditions (1.15), (1.16) and (1.17). In particular, if (1.3) and (1.4) are satisfied, one can take $\Gamma:=K / \eta$ in (1.16). On the other hand, as it will be apparent in Appendix A, it is technically convenient to avoid requesting assumption (1.3) in order not to limit the potential of the general approach that we present.
} 
We remark that $\gamma_{2}=\gamma_{3}=0$, and thus $\gamma=\gamma_{1}$, when $a$ depends only on $t$ and $u$ (but is independent of the space variable), and $H$ depends only on $x, t$ and $u$ (but is independent of the gradient and of the Hessian of the solution).

Given $\delta \in(0, T)$ and $\rho \in(0, R)$, we define

$$
\begin{aligned}
& \mathcal{C}:=\sqrt{\mu}+\sqrt[3]{\gamma} \\
\mathcal{T} & :=\frac{1}{\sqrt{\delta}} \\
\text { and } \quad \mathcal{S} & :=\frac{1}{\rho}+\frac{1}{\sqrt{\rho(R-\rho)}}+\frac{\sqrt[4]{k_{+}}}{\sqrt{\rho}} .
\end{aligned}
$$

We notice that $\mathcal{C}, \mathcal{T}$ and $\mathcal{S}$ are functions of $(x, t)$. Moreover, we set

$$
\begin{aligned}
\tau_{u} & :=\sup _{x \in B\left(x_{0}, R\right)} \frac{F^{\prime}(u)|\nabla u|}{u(\xi-G(u))}\left(x, t_{0}-T\right), \\
\text { and } \quad \sigma_{u} & :=\sup _{\substack{x \in \partial B\left(x_{0}, R\right) \\
t \in\left[t_{0}-T, t_{0}\right]}} \frac{F^{\prime}(u)|\nabla u|}{u(\xi-G(u))}(x, t) .
\end{aligned}
$$

We also consider the functions

$$
\begin{array}{ll} 
& \mathcal{B}_{1}(x, t):=\chi_{B\left(x_{0}, R-\rho\right)}(x) \chi_{\left[t_{0}-T, t_{0}-T+\delta\right)}(t), \\
& \mathcal{B}_{2}(x, t):=\chi_{B\left(x_{0}, R\right) \backslash B\left(x_{0}, R-\rho\right)}(x) \chi_{\left[t_{0}-T+\delta, t_{0}\right]}(t), \\
& \mathcal{B}_{3}(x, t):=\chi_{B\left(x_{0}, R\right) \backslash B\left(x_{0}, R-\rho\right)}(x) \chi_{\left[t_{0}-T, t_{0}-T+\delta\right)}(t) \\
\text { and } \quad & \mathcal{J}(x, t):=\chi_{B\left(x_{0}, R-\rho\right)}(x) \chi_{\left[t_{0}-T+\delta, t_{0}\right]}(t) .
\end{array}
$$

As customary, we used here the standard notation for the characteristic function of a set $S$, that is

$$
\chi_{S}(z):= \begin{cases}1 & \text { if } z \in S, \\ 0 & \text { otherwise }\end{cases}
$$

Also, given a constant $C>0$ (to be appropriately chosen conveniently large in the following) we define

$$
\begin{aligned}
\beta_{1} & :=\tau_{u}+\min \left\{\sigma_{u}, C \mathcal{S}\right\}, \\
\beta_{2} & :=\sigma_{u}+\min \left\{\tau_{u}, C \mathcal{T}\right\}, \\
\beta_{3} & :=\sigma_{u}+\tau_{u} \\
\text { and } \quad \iota & :=\min \left\{\sigma_{u}+\tau_{u}, C(\mathcal{T}+\mathcal{S})\right\} .
\end{aligned}
$$

Let also

$$
z:=\beta_{1} \mathcal{B}_{1}+\beta_{2} \mathcal{B}_{2}+\beta_{3} \mathcal{B}_{3}+\iota \mathcal{J}
$$

With this notation, the main result of this paper is the following global gradient estimate, valid in all the domain where a parabolic nonlinear equation holds true:

Theorem 1.5. Let $\mathcal{M}$ be a complete Riemannian manifold of dimension n satisfying (1.9). Let a $\in C^{1}\left(Q_{R, T} \times \mathbb{R}\right)$ satisfying (1.10) and $F \in C^{2}(0,+\infty)$ satisfying (1.11), (1.12), (1.15), (1.16) and (1.17).

Let $u$ be a positive, bounded and smooth solution of the evolution equation (1.8) in $Q_{R, T}$. 
Then, there exists a constant $C>0$ depending only on $n, \Gamma, \kappa, a_{0}$ and $\eta$ such that the following estimate holds true:

$$
G^{\prime}(u(x, t))|\nabla u(x, t)| \leq(C \mathcal{C}+z(x, t))(\xi-G(u(x, t))) \quad \text { for all }(x, t) \in Q_{R, T} .
$$

Here we used the notation in (1.14), (1.25) and (1.29).

Interestingly, Theorem 1.5 includes several recent results in the literature as a special case. For instance, the particular choice

$$
F(s):=s, a:=1 \text { and } H:=H(x, t, u)
$$

corresponding to the equation $\partial_{t} u=\Delta u+H(x, t, u)$, taking $\xi:=1, \eta:=1, s_{0}:=M$, produces in (1.18) the quantity

$$
\mu_{1}=\sup _{(x, t) \in Q_{R, T}}\left(k+\partial_{u} H(x, t, u(x, t))-\frac{H(x, t, u(x, t))}{u(x, t)}+\frac{H(x, t, u(x, t))}{\left(1+\log \frac{M}{u(x, t)}\right) u(x, t)}\right)_{+}
$$

which coincides with the quantity in (1.6) of [10]; similarly, in such a case, in (1.19) we find

$$
\gamma_{1}=\sup _{(x, t) \in Q_{R, T}} \frac{|\nabla H(x, t, u(x, t))|}{u(x, t)},
$$

which coincides with the quantity in (1.4) of [10]; also, in (1.20), (1.22) and (1.23) one finds $\mu_{2}=\gamma_{2}=\gamma_{3}=0$, therefore Theorem 1.5 here recovers, in the special setting of (1.30), the result given in Theorem 1.1 of [10].

Differently from the previous literature, our general framework comprises, as a particular case, the equation $u_{t}=u \Delta u+g(u)$ which models the spread of an epidemic in a closed population without remotion and is often used as a prototype for complicated and sometimes pathological behavior of the solutions, see [11, 26].

It is also interesting to comment on the structure of the estimate obtained in Theorem 1.5, and especially on the dependence of the bound obtained by the quantities $\mu$ and $\gamma$. Specifically, being a gradient estimate, one would like the terms on the right hand side of the estimate to be independent of the derivatives of the solution, while, at a first glance, these quantities may depend on the derivatives up to order three. Nevertheless:

- The dependence of $\mu_{1}$ and $\gamma_{1}$ in (1.18) and (1.19) on the derivatives of $u$ only occurs via the source term $H$ : in particular, if $H$ and its derivatives are uniformly bounded, then $\mu_{1}$ and $\gamma_{1}$ can be bounded independently on the derivatives of $u$;

- The quantities $\mu_{2}$ and $\gamma_{2}$ in (1.20) and (1.22) depend on the derivatives of the solution up to the second order, but they vanish if the diffusion coefficient $a$ is either constant or depends only on time;

- The quantity $\gamma_{3}$ in (1.23) depends on the derivatives up to the third order of the solution, but it vanishes if the source term $H$ does not depend on the derivatives of the solution.

That is: on the one hand, in its general form, under additional bounds on the derivatives of the solution, the estimate in Theorem 1.5 can be considered as a pointwise estimate at any $(x, t) \in Q_{R, T}$; on the other hand, for the special (but still extremely general) case given by the equation

$$
\partial_{t} u=a(t) \Delta(F(u))+H(x, t, u),
$$


then the structural quantities $\mu$ and $\gamma$ can be bounded independently from the derivatives of the solution, reducing to

$$
\begin{aligned}
& \mu= \sup _{(x, t) \in Q_{R, T}}\left(k a(t) F^{\prime}(u(x, t))+\frac{H(x, t, u(x, t)) F^{\prime \prime}(u(x, t))}{F^{\prime}(u(x, t))}\right. \\
&\left.+\partial_{u} H(x, t, u(x, t))-\frac{H(x, t, u(x, t))}{u(x, t)}+\frac{H(x, t, u(x, t)) F^{\prime}(u(x, t))}{(\xi-G(u(x, t))) u(x, t)}\right)_{+} \\
& \text {and } \quad \gamma=\sup _{(x, t) \in Q_{R, T}} \frac{F^{\prime}(u(x, t))|\nabla H(x, t, u(x, t))|}{u(x, t)} .
\end{aligned}
$$

The relevant structural quantities in case of an equation of the type

$$
\partial_{t} u=a(t) \Delta u^{p}+\varepsilon|\nabla u|^{q}
$$

with $\varepsilon>0$ will be discussed, as an exemplifying situation, in Appendix B.

Furthermore, one deduces from the global estimate of Theorem 1.5 a local estimate in $Q_{R / 2, T / 2}$, according to the following result.

Corollary 1.6. Let $\mathcal{M}$ be a complete Riemannian manifold of dimension $n$ satisfying (1.9). Let a $\in C^{1}\left(Q_{R, T} \times\right.$ $\mathbb{R})$ satisfying $(1.10)$ and $F \in C^{2}(0,+\infty)$ satisfying (1.11), (1.12), (1.15), (1.16) and (1.17).

Let $u$ be a positive, bounded and smooth solution of the evolution equation (1.8) in $Q_{R, T}$.

Then, there exists a constant $C>0$ depending only on $n, \Gamma, \kappa, a_{0}$ and $\eta$ such that the following estimate holds true: for every $(x, t) \in Q_{R / 2, T / 2}$,

$$
G^{\prime}(u(x, t))|\nabla u(x, t)| \leq C\left(\sqrt{\mu}+\sqrt[3]{\gamma}+\frac{1}{R}+\frac{1}{\sqrt{T}}+\frac{\sqrt[4]{k_{+}}}{\sqrt{R}}\right)(\xi-G(u(x, t)))
$$

Here we used the notation in (1.14), (1.21) and (1.24).

We also stress that when $H:=0$ and $a:=1$, then $\mu=k_{+}$and $\gamma=0$, therefore Corollary 1.6 contains Theorem 1.3 (that is, Thm. 7 in [22]) as a special case. In addition, it also contains Corollary 1.4 (that is, Cor. 9 in [22]) as a particular subcase, as observed in Appendix A.

It is also interesting to compare the statements of Theorem 1.5 and Corollary 1.6. Evidently, the estimate obtained in Theorem 1.5 is global, since it is valid in the whole of the domain where the equation is satisfied. For this, the estimate obtained in Theorem 1.5 necessarily must take into account the "parabolic data" of the equation, which are encoded in the quantities $\tau_{u}$ and $\sigma_{u}$ defined in (1.26). On the other hand, the estimate obtained in Corollary 1.6 holds true only in a subdomain, but then it becomes independent of the "parabolic data" of the equation and relies only on the structural functions of the equation and on the geometry of the domain.

We emphasize that the general estimate in Theorem 1.5 is stronger than the one in Corollary 1.6 even if one reduces to $Q_{R / 2, T / 2}$, since one can also deduce from it that, in $Q_{R / 2, T / 2}$,

$$
G^{\prime}(u(x, t))|\nabla u(x, t)| \leq C\left(\sqrt{\mu}+\sqrt[3]{\gamma}+\sigma_{u}+\tau_{u}\right)(\xi-G(u(x, t)))
$$

which is a sharper estimate than the one in (1.32) when the data of the equation are particularly convenient to make $\tau_{u}$ and $\sigma_{u}$ sufficiently small. That is, while the estimate in Corollary 1.6 has the advantages of being easier to read and "universal" (i.e., not depending on the boundary data of the equation), the estimate in Theorem 1.5 is more precise, since it allows one to possibly recall the boundary data in order to achieve a sharper result. 
In any case, to the best of our knowledge, Theorem 1.5 is the first global estimate for nonlinear parabolic operators, even in the case of porous medium equation with no source terms, and also the local version in Corollary 1.6 is the first local estimate to take into account general porous medium equations with source terms; besides, the alternative estimate in (1.33) is the first occurrence in which an improved estimate for these parabolic equations driven by nonlinear operators is obtained thanks to the boundary data. Moreover, the results obtained are new even in the Euclidean setting.

We also remark that suitable Liouville-type results can be easily deduced from our main estimates: as an example, we provide a rigidity result in Appendix $\mathrm{C}$ that relies on Corollary 1.6. See also Theorems 1.3 and 1.5 for related Liouville-type results for porous media equations.

The rest of this paper is organized as follows. Section 2 presents the computations related to a suitable auxiliary function that will be used to deduce the main results from the maximum principle. In Section 3 we develop the necessary calculations to localize the problem by using suitable cut-off functions in space and time. Section 4 contains the proof of Theorem 1.5 and Section 5 provides the one of Corollary 1.6.

\section{AN AUXILIARY BARRIER}

A common procedure in the theory of elliptic and parabolic equations is to introduce a suitable auxiliary function (that will be denoted by $w$ in our context) which satisfies a convenient equation; with this, an appropriate use of the maximum principle provides estimates on the auxiliary function, which can be traced back to the original solution. To implement this technique in our framework, we argue as follows.

Given $G$ as in (1.14), for all $r \in \mathbb{R}$ we define

$$
g(r):=G\left(e^{r}\right)
$$

and

$$
\lambda(r):=\frac{g^{\prime}(r)}{\xi-g(r)}-1+\frac{\sqrt{n}\left|g^{\prime \prime}(r)\right|}{2 g^{\prime}(r)} .
$$

It is interesting to observe that, by (1.14),

$$
G^{\prime}(r)=\frac{F^{\prime}(r)}{r} \quad \text { and } \quad G^{\prime \prime}(r)=\frac{F^{\prime \prime}(r)}{r}-\frac{F^{\prime}(r)}{r^{2}}
$$

In addition, since, by (2.1), we know that $g^{\prime}(r)=e^{r} G^{\prime}\left(e^{r}\right)$, we deduce from the assumption (1.11) on $F^{\prime}$ and (2.3) that

$$
g^{\prime}(r)=F^{\prime}\left(e^{r}\right)>0 \quad \text { and } \quad g^{\prime \prime}(r)=e^{r} F^{\prime \prime}\left(e^{r}\right) .
$$

Also, given $u$ as in the statement of Theorem 1.5, we set

$$
v(x, t):=\ln u(x, t)
$$

and

$$
w(x, t):=\mid \nabla \ln \left(\xi-\left.g(v(x, t))\right|^{2}=\frac{\mid \nabla\left(\left.g(v(x, t))\right|^{2}\right.}{(\xi-g(v(x, t)))^{2}} .\right.
$$

We stress that $w$ is well defined, since

$$
\xi-g(v(x, t))=\xi-G(u(x, t)) \geq \eta>0,
$$


thanks to (1.15).

Also, as usual, the notation $\left(u, \nabla u, D^{2} u\right)$ will be used as short for $\left(u(x, t), \nabla u(x, t), D^{2} u(x, t)\right)$. Furthermore, the notation $g^{\prime}$ stands for the derivative of $g(r)$ with respect to $r$, hence $g^{\prime}(v)$ is a short notation for $g^{\prime}(v(x, t))$. To clarify this framework, let us point out that

$$
\begin{aligned}
& \nabla\left(g(v(x, t))=g^{\prime}(v(x, t)) \nabla v(x, t)\right. \\
& \text { and } \quad \nabla\left(g^{\prime}(v(x, t))=g^{\prime \prime}(v(x, t)) \nabla v(x, t)=\frac{g^{\prime \prime}(v(x, t)) \nabla(g(v(x, t))}{g^{\prime}(v(x, t))}\right. \text {. }
\end{aligned}
$$

We recall that the latter denominator is nonzero, thanks to (2.4). With this setting, we can state the main result of this section as follows:

Lemma 2.1. Let $u$ be as in Theorem 1.5. Then, in $Q_{R, T}$,

$$
\frac{a g^{\prime} \Delta w-w_{t}}{2} \geq a \kappa(\xi-g) w^{2}+a \lambda\langle\nabla w, \nabla g\rangle-\mu w-\frac{\gamma|\nabla g|}{(\xi-g)^{2}}
$$

where $g$ is a short notation for $g(v(x, t))$. Here, $\kappa$ and $\xi$ are given in assumptions (1.12) and (1.15), $\lambda$ in (2.2), $\mu$ in (1.21) and $\gamma$ in (1.24).

Proof. We note that

$$
\Delta(F(u))=F^{\prime}(u) \Delta u+F^{\prime \prime}(u)|\nabla u|^{2} .
$$

Hence, by (1.8),

$$
u_{t}=a F^{\prime}(u) \Delta u+a F^{\prime \prime}(u)|\nabla u|^{2}+H .
$$

Also, by (2.5),

$$
v_{t}=\frac{u_{t}}{u} \quad \text { and } \quad \nabla v=\frac{\nabla u}{u},
$$

whence

$$
\frac{G^{\prime}(u)}{u}|\nabla u|^{2}=\frac{\nabla(G(u)) \cdot \nabla u}{u}=\nabla(G(u)) \cdot \nabla v .
$$

To ease the notation, we write $\nabla G$ as a short notation for $\nabla(G(u))=\nabla(G(u(x, t)))$ (of course, no confusion should arise with $\nabla G(u(x, t)))$. Accordingly, exploiting (2.3), (2.10), (2.11), (2.12) and (2.13),

$$
\begin{aligned}
v_{t} & =\frac{a F^{\prime}(u) \Delta u+a F^{\prime \prime}(u)|\nabla u|^{2}+H}{u} \\
& =a G^{\prime}(u) \Delta u+a\left(G^{\prime \prime}(u)+\frac{F^{\prime}(u)}{u^{2}}\right)|\nabla u|^{2}+\frac{H}{u} \\
& =a G^{\prime}(u) \Delta u+a\left(G^{\prime \prime}(u)+\frac{G^{\prime}(u)}{u}\right)|\nabla u|^{2}+\frac{H}{u} \\
& =a \Delta(G(u))+a\langle\nabla G, \nabla v\rangle+\frac{H}{u} .
\end{aligned}
$$


Thus, recalling (2.1) and (2.5), we can write $G(u)=g(v)$ and thereby obtain that

$$
v_{t}=a \Delta(g(v))+a\langle\nabla g, \nabla v\rangle+\frac{H}{u} .
$$

where $\nabla g$ is short for $\nabla(g(v))=\nabla(g(v(x, t)))$.

To ease the notation, we also write $g_{t}$ to mean $\partial_{t}(g(v))$. As a consequence, by $(2.14)$ we have that

$$
\begin{aligned}
g_{t}=g^{\prime}(v) v_{t} & =a g^{\prime}(v) \Delta(g(v))+a\left\langle\nabla g, g^{\prime}(v) \nabla v\right\rangle+\frac{g^{\prime}(v) H}{u} \\
& =a g^{\prime}(v) \Delta(g(v))+a|\nabla g|^{2}+\frac{g^{\prime}(v) H}{u}
\end{aligned}
$$

Now we observe that, by (2.6),

$$
\nabla w=\frac{\nabla|\nabla g|^{2}}{(\xi-g)^{2}}+2 \frac{|\nabla g|^{2} \nabla g}{(\xi-g)^{3}}
$$

Moreover, we have that

$$
\operatorname{div}\left(\frac{\nabla|\nabla g|^{2}}{(\xi-g)^{2}}\right)=\frac{\Delta|\nabla g|^{2}}{(\xi-g)^{2}}+\frac{2\left\langle\nabla|\nabla g|^{2}, \nabla g\right\rangle}{(\xi-g)^{3}}
$$

In addition,

$$
\operatorname{div}\left(\frac{|\nabla g|^{2} \nabla g}{(\xi-g)^{3}}\right)=\frac{\left\langle\nabla|\nabla g|^{2}, \nabla g\right\rangle}{(\xi-g)^{3}}+\frac{|\nabla g|^{2} \Delta g}{(\xi-g)^{3}}+\frac{3|\nabla g|^{4}}{(\xi-g)^{4}}
$$

From this, (2.16) and (2.17), we deduce that

$$
\begin{aligned}
& \Delta w \\
= & \frac{\Delta|\nabla g|^{2}}{(\xi-g)^{2}}+\frac{2\left\langle\nabla|\nabla g|^{2}, \nabla g\right\rangle}{(\xi-g)^{3}}+\frac{2\left\langle\nabla|\nabla g|^{2}, \nabla g\right\rangle}{(\xi-g)^{3}}+\frac{2|\nabla g|^{2} \Delta g}{(\xi-g)^{3}}+\frac{6|\nabla g|^{4}}{(\xi-g)^{4}} \\
= & \frac{\Delta|\nabla g|^{2}}{(\xi-g)^{2}}+\frac{4\left\langle\nabla|\nabla g|^{2}, \nabla g\right\rangle}{(\xi-g)^{3}}+\frac{2|\nabla g|^{2} \Delta g}{(\xi-g)^{3}}+\frac{6|\nabla g|^{4}}{(\xi-g)^{4}} .
\end{aligned}
$$

Besides, using (2.6) and (2.15), we find that

$$
\begin{aligned}
w_{t}= & \frac{2\left\langle\nabla g, \nabla g_{t}\right\rangle}{(\xi-g)^{2}}+\frac{2|\nabla g|^{2} g_{t}}{(\xi-g)^{3}} \\
= & \frac{2\left\langle\nabla g, \nabla\left(a g^{\prime} \Delta g\right)\right\rangle}{(\xi-g)^{2}}+\frac{2\left\langle\nabla g, \nabla\left(a|\nabla g|^{2}\right)\right\rangle}{(\xi-g)^{2}}+\frac{2\left\langle\nabla g, \nabla\left(\frac{g^{\prime}(v) H}{u}\right)\right\rangle}{(\xi-g)^{2}}+\frac{2 a g^{\prime} \Delta g|\nabla g|^{2}}{(\xi-g)^{3}} \\
& +\frac{2 a|\nabla g|^{4}}{(\xi-g)^{3}}+\frac{2|\nabla g|^{2}\left(\frac{g^{\prime}(v) H}{u}\right)}{(\xi-g)^{3}} .
\end{aligned}
$$


In light of (2.8), we also have that

$$
\begin{aligned}
& \left\langle\nabla g, \nabla\left(a g^{\prime} \Delta g\right)\right\rangle \\
= & a g^{\prime}\langle\nabla g, \nabla \Delta g\rangle+a \Delta g\left\langle\nabla g, \nabla g^{\prime}\right\rangle+g^{\prime} \Delta g\langle\nabla g, \nabla a\rangle+g^{\prime} \Delta g\left\langle\nabla g, \partial_{u} a \nabla u\right\rangle \\
= & a g^{\prime}\langle\nabla g, \nabla \Delta g\rangle+\frac{a g^{\prime \prime} \Delta g}{g^{\prime}}|\nabla g|^{2}+g^{\prime} \Delta g\langle\nabla g, \nabla a\rangle+\partial_{u} a g^{\prime} \Delta g\langle\nabla g, \nabla u\rangle .
\end{aligned}
$$

Now, using (2.4) and (2.5), we see that $g^{\prime \prime}(v)=F^{\prime \prime}(u) u$. Thus, recalling the coordinate notation in (1.13), and making also use of (2.8) and (2.12), we find that

$$
\begin{aligned}
\nabla\left(\frac{g^{\prime}(v) H}{u}\right) & =\frac{g^{\prime \prime}(v) H \nabla v}{u}+\frac{g^{\prime}(v) \nabla H}{u}+\frac{g^{\prime}(v) \partial_{u} H \nabla u}{u}-\frac{g^{\prime}(v) H \nabla u}{u^{2}}+\Upsilon_{1} \\
& =\frac{F^{\prime \prime}(u) H \nabla g}{F^{\prime}(u)}+\frac{F^{\prime}(u) \nabla H}{u}+\partial_{u} H \nabla g-\frac{H \nabla g}{u}+\Upsilon_{1}
\end{aligned}
$$

where

$$
\Upsilon_{1}:=\frac{g^{\prime}(v)}{u}\left(\sum_{i=1}^{n} \partial_{\omega_{i}} H \nabla \partial_{x_{i}} u+\sum_{i, j=1}^{n} \partial_{\Omega_{i j}} H \nabla \partial_{x_{i} x_{j}}^{2} u\right)
$$

This observation, together with (2.19), (2.20) and (2.21), yields that

$$
\begin{aligned}
w_{t}= & \frac{2 a g^{\prime}\langle\nabla g, \nabla \Delta g\rangle}{(\xi-g)^{2}}+\frac{\frac{2 a g^{\prime \prime} \Delta g}{g^{\prime}}|\nabla g|^{2}}{(\xi-g)^{2}}+\frac{2\left\langle\nabla g, \nabla\left(a|\nabla g|^{2}\right)\right\rangle}{(\xi-g)^{2}}+\frac{2 F^{\prime \prime}(u) H|\nabla g|^{2}}{F^{\prime}(u)(\xi-g)^{2}} \\
& +\frac{2 F^{\prime}(u)\langle\nabla g, \nabla H\rangle}{u(\xi-g)^{2}}+\frac{2 \partial_{u} H|\nabla g|^{2}}{(\xi-g)^{2}}-\frac{2 H|\nabla g|^{2}}{u(\xi-g)^{2}} \\
& +\frac{2 a g^{\prime} \Delta g|\nabla g|^{2}}{(\xi-g)^{3}}+\frac{2 a|\nabla g|^{4}}{(\xi-g)^{3}}+\frac{2|\nabla g|^{2}\left(\frac{g^{\prime}(v) H}{u}\right)}{(\xi-g)^{3}}+\Upsilon_{2} \\
= & \frac{2 a g^{\prime}\langle\nabla g, \nabla \Delta g\rangle}{(\xi-g)^{2}}+\frac{2 a g^{\prime \prime} \Delta g|\nabla g|^{2}}{g^{\prime}(\xi-g)^{2}}+\frac{2\left\langle\nabla g, \nabla\left(a|\nabla g|^{2}\right)\right\rangle}{(\xi-g)^{2}}+\frac{2 F^{\prime \prime}(u) H|\nabla g|^{2}}{F^{\prime}(u)(\xi-g)^{2}} \\
& +\frac{2 F^{\prime}(u)\langle\nabla g, \nabla H\rangle}{u(\xi-g)^{2}}+\frac{2 \partial_{u} H|\nabla g|^{2}}{(\xi-g)^{2}}-\frac{2 H|\nabla g|^{2}}{u(\xi-g)^{2}} \\
& +\frac{2 a g^{\prime} \Delta g|\nabla g|^{2}}{(\xi-g)^{3}}+\frac{2 a|\nabla g|^{4}}{(\xi-g)^{3}}+\frac{2 F^{\prime}(u) H|\nabla g|^{2}}{u(\xi-g)^{3}}+\Upsilon_{2},
\end{aligned}
$$

where

$$
\Upsilon_{2}:=\frac{2\left\langle\nabla g, \Upsilon_{1}\right\rangle}{(\xi-g)^{2}}+\frac{2 g^{\prime} \Delta g\left\langle\nabla g, \nabla a+\partial_{u} a \nabla u\right\rangle}{(\xi-g)^{2}}
$$


This and (2.18), after the cancellation of the term $\frac{2 a g^{\prime} \Delta g|\nabla g|^{2}}{(\xi-g)^{3}}$, give that

$$
\begin{aligned}
a g^{\prime} \Delta w-w_{t}= & \frac{a g^{\prime} \Delta|\nabla g|^{2}}{(\xi-g)^{2}}+\frac{4 a g^{\prime}\left\langle\nabla|\nabla g|^{2}, \nabla g\right\rangle}{(\xi-g)^{3}}+\frac{6 a g^{\prime}|\nabla g|^{4}}{(\xi-g)^{4}}-\frac{2 a g^{\prime}\langle\nabla g, \nabla \Delta g\rangle}{(\xi-g)^{2}} \\
& -\frac{2 F^{\prime \prime}(u) H|\nabla g|^{2}}{F^{\prime}(u)(\xi-g)^{2}}-\frac{2 F^{\prime}(u)\langle\nabla g, \nabla H\rangle}{u(\xi-g)^{2}}-\frac{2 \partial_{u} H|\nabla g|^{2}}{(\xi-g)^{2}} \\
& +\frac{2 H|\nabla g|^{2}}{u(\xi-g)^{2}}-\frac{2 a g^{\prime \prime} \Delta g|\nabla g|^{2}}{g^{\prime}(\xi-g)^{2}}-\frac{2\left\langle\nabla g, \nabla\left(a|\nabla g|^{2}\right)\right\rangle}{(\xi-g)^{2}}-\frac{2 a|\nabla g|^{4}}{(\xi-g)^{3}} \\
& -\frac{2 F^{\prime}(u) H|\nabla g|^{2}}{u(\xi-g)^{3}}-\Upsilon_{2} .
\end{aligned}
$$

Now we recall the Bochner's formula, according to which

$$
\Delta\left(\frac{|\nabla g|^{2}}{2}\right)=\langle\nabla \Delta g, \nabla g\rangle+\left|D^{2} g\right|^{2}+\operatorname{Ric}(\nabla g, \nabla g) .
$$

This and the Ricci curvature assumption in (1.9) entail that

$$
\Delta|\nabla g|^{2}-2\langle\nabla \Delta g, \nabla g\rangle=2\left|D^{2} g\right|^{2}+2 \operatorname{Ric}(\nabla g, \nabla g) \geq 2\left|D^{2} g\right|^{2}-2 k|\nabla g|^{2},
$$

where, as customary, the norm of a matrix is taken to be the square root of the sum of the squares of its entry.

Plugging this information in (2.24), we conclude that

$$
\begin{aligned}
a g^{\prime} \Delta w-w_{t} \geq & \frac{2 a g^{\prime}\left|D^{2} g\right|^{2}}{(\xi-g)^{2}}-\frac{2 a g^{\prime} k|\nabla g|^{2}}{(\xi-g)^{2}}+\frac{4 a g^{\prime}\left\langle\nabla|\nabla g|^{2}, \nabla g\right\rangle}{(\xi-g)^{3}}+\frac{6 a g^{\prime}|\nabla g|^{4}}{(\xi-g)^{4}} \\
& -\frac{2 F^{\prime \prime}(u) H|\nabla g|^{2}}{F^{\prime}(u)(\xi-g)^{2}}-\frac{2 F^{\prime}(u)\langle\nabla g, \nabla H\rangle}{u(\xi-g)^{2}}-\frac{2 \partial_{u} H|\nabla g|^{2}}{(\xi-g)^{2}} \\
& +\frac{2 H|\nabla g|^{2}}{u(\xi-g)^{2}}-\frac{2 a g^{\prime \prime} \Delta g|\nabla g|^{2}}{g^{\prime}(\xi-g)^{2}}-\frac{2\left\langle\nabla g, \nabla\left(a|\nabla g|^{2}\right)\right\rangle}{(\xi-g)^{2}}-\frac{2 a|\nabla g|^{4}}{(\xi-g)^{3}} \\
& -\frac{2 F^{\prime}(u) H|\nabla g|^{2}}{u(\xi-g)^{3}}-\Upsilon_{2} \\
\geq & \frac{2 a g^{\prime}\left|D^{2} g\right|^{2}}{(\xi-g)^{2}}+\frac{4 a g^{\prime}\left\langle\nabla|\nabla g|^{2}, \nabla g\right\rangle}{(\xi-g)^{3}}+\frac{6 a g^{\prime}|\nabla g|^{4}}{(\xi-g)^{4}}-\frac{2 \mu_{1}|\nabla g|^{2}}{(\xi-g)^{2}} \\
& -\frac{2 \gamma_{1}|\nabla g|}{(\xi-g)^{2}}-\frac{2 a g^{\prime \prime} \Delta g|\nabla g|^{2}}{g^{\prime}(\xi-g)^{2}}-\frac{2\left\langle\nabla g, \nabla\left(a|\nabla g|^{2}\right)\right\rangle}{(\xi-g)^{2}}-\frac{2 a|\nabla g|^{4}}{(\xi-g)^{3}}-\Upsilon_{2},
\end{aligned}
$$

where the definitions of $\mu_{1}$ and $\gamma_{1}$ in (1.18) and (1.19) have been exploited.

It is now convenient to define

$$
\zeta:=\left(\sqrt{n} \frac{\left|g^{\prime \prime}\right|}{g^{\prime}}(\xi-g)\right)^{-1}
$$


We point out that $\zeta \in(0,+\infty]$, due to (2.4) and (2.7). By the Cauchy-Schwarz inequality, if $g^{\prime \prime} \neq 0$ (hence $\zeta \neq$ $+\infty)$, one has that

$$
2\left|\frac{g^{\prime \prime} \Delta g|\nabla g|^{2}}{g^{\prime}(\xi-g)^{2}}\right| \leq \zeta\left(\frac{g^{\prime \prime}}{g^{\prime}}\right)^{2}(\Delta g)^{2}+\frac{|\nabla g|^{4}}{\zeta(\xi-g)^{4}} \leq \zeta n\left(\frac{g^{\prime \prime}}{g^{\prime}}\right)^{2}\left|D^{2} g\right|^{2}+\frac{|\nabla g|^{4}}{\zeta(\xi-g)^{4}} .
$$

We will now make use of (2.27) at all points, with the convention that, since the left hand side of (2.27) vanishes when $g^{\prime \prime}=0$, the terms involving $\zeta$ can simply be neglected in the forthcoming computations. In this sense, putting together (2.25) and (2.27) we see that

$$
\begin{aligned}
a g^{\prime} \Delta w-w_{t} \geq[ & \left.2 g^{\prime}-\zeta n\left(\frac{g^{\prime \prime}}{g^{\prime}}\right)^{2}(\xi-g)^{2}\right] \frac{a\left|D^{2} g\right|^{2}}{(\xi-g)^{2}}+\frac{4 a g^{\prime}\left\langle\nabla|\nabla g|^{2}, \nabla g\right\rangle}{(\xi-g)^{3}} \\
& +\left(6 g^{\prime}-\frac{1}{\zeta}\right) \frac{a|\nabla g|^{4}}{(\xi-g)^{4}}-\frac{2\left\langle\nabla g, \nabla\left(a|\nabla g|^{2}\right)\right\rangle}{(\xi-g)^{2}} \\
& -\frac{2 a|\nabla g|^{4}}{(\xi-g)^{3}}-\frac{2 \mu_{1}|\nabla g|^{2}}{(\xi-g)^{2}}-\frac{2 \gamma_{1}|\nabla g|}{(\xi-g)^{2}}-\Upsilon_{2} .
\end{aligned}
$$

Furthermore, in light of (2.16),

$$
\langle\nabla w, \nabla g\rangle=\frac{\left\langle\nabla|\nabla g|^{2}, \nabla g\right\rangle}{(\xi-g)^{2}}+\frac{2|\nabla g|^{4}}{(\xi-g)^{3}}
$$

and, as a result,

$$
\begin{aligned}
& \frac{4 a g^{\prime}\left\langle\nabla|\nabla g|^{2}, \nabla g\right\rangle}{(\xi-g)^{3}}+\frac{6 a g^{\prime}|\nabla g|^{4}}{(\xi-g)^{4}} \\
= & \left(\frac{2 a g^{\prime}\left\langle\nabla|\nabla g|^{2}, \nabla g\right\rangle}{(\xi-g)^{3}}+\frac{4 a g^{\prime}|\nabla g|^{4}}{(\xi-g)^{4}}\right)+\left(\frac{2 a g^{\prime}\left\langle\nabla|\nabla g|^{2}, \nabla g\right\rangle}{(\xi-g)^{3}}+\frac{2 a g^{\prime}|\nabla g|^{4}}{(\xi-g)^{4}}\right) \\
= & \frac{2 a g^{\prime}\langle\nabla w, \nabla g\rangle}{\xi-g}+\frac{2 a g^{\prime}\left\langle\nabla|\nabla g|^{2}, \nabla g\right\rangle}{(\xi-g)^{3}}+\frac{2 a g^{\prime}|\nabla g|^{4}}{(\xi-g)^{4}} .
\end{aligned}
$$

The previous two identities, combined with (2.28), yield that

$$
\begin{aligned}
a g^{\prime} \Delta w-w_{t} \geq & {\left[2 g^{\prime}-\zeta n\left(\frac{g^{\prime \prime}}{g^{\prime}}\right)^{2}(\xi-g)^{2}\right] \frac{a\left|D^{2} g\right|^{2}}{(\xi-g)^{2}}+\frac{2 a g^{\prime}\left\langle\nabla|\nabla g|^{2}, \nabla g\right\rangle}{(\xi-g)^{3}} } \\
& +\left(2 g^{\prime}-\frac{1}{\zeta}\right) \frac{a|\nabla g|^{4}}{(\xi-g)^{4}}+\frac{2 a|\nabla g|^{4}}{(\xi-g)^{3}}+2 a\left(\frac{g^{\prime}}{\xi-g}-1\right)\langle\nabla w, \nabla g\rangle \\
& -\frac{2 \mu_{1}|\nabla g|^{2}}{(\xi-g)^{2}}-\frac{2 \gamma_{1}|\nabla g|}{(\xi-g)^{2}}-\Upsilon_{3},
\end{aligned}
$$

where

$$
\Upsilon_{3}:=\Upsilon_{2}+\frac{2\left\langle\nabla g, \nabla a+\partial_{u} a \nabla u\right\rangle|\nabla g|^{2}}{(\xi-g)^{2}}
$$


It is now convenient to factor out a term of the type

$$
\Xi:=2 g^{\prime}-\zeta n\left(\frac{g^{\prime \prime}}{g^{\prime}}\right)^{2}(\xi-g)^{2}
$$

from the first three terms in the right hand side of (2.29) (up to a reminder). For this, we write

$$
\begin{gathered}
{\left[2 g^{\prime}-\zeta n\left(\frac{g^{\prime \prime}}{g^{\prime}}\right)^{2}(\xi-g)^{2}\right] \frac{a\left|D^{2} g\right|^{2}}{(\xi-g)^{2}}+\frac{2 a g^{\prime}\left\langle\nabla|\nabla g|^{2}, \nabla g\right\rangle}{(\xi-g)^{3}}+\left(2 g^{\prime}-\frac{1}{\zeta}\right) \frac{a|\nabla g|^{4}}{(\xi-g)^{4}}} \\
=\Xi \frac{a\left|D^{2} g\right|^{2}}{(\xi-g)^{2}}+\left(\Xi+\zeta n\left(\frac{g^{\prime \prime}}{g^{\prime}}\right)^{2}(\xi-g)^{2}\right) \frac{a\left\langle\nabla|\nabla g|^{2}, \nabla g\right\rangle}{(\xi-g)^{3}} \\
\quad+\left(\Xi+\zeta n\left(\frac{g^{\prime \prime}}{g^{\prime}}\right)^{2}(\xi-g)^{2}-\frac{1}{\zeta}\right) \frac{a|\nabla g|^{4}}{(\xi-g)^{4}} \\
=\Xi\left(\frac{a\left|D^{2} g\right|^{2}}{(\xi-g)^{2}}+\frac{a\left\langle\nabla|\nabla g|^{2}, \nabla g\right\rangle}{(\xi-g)^{3}}+\frac{a|\nabla g|^{4}}{(\xi-g)^{4}}\right) \\
\quad+\zeta n\left(\frac{g^{\prime \prime}}{g^{\prime}}\right)^{2} \frac{a\left\langle\nabla|\nabla g|^{2}, \nabla g\right\rangle}{\xi-g}+\left(\zeta n\left(\frac{g^{\prime \prime}}{g^{\prime}}\right)^{2}(\xi-g)^{2}-\frac{1}{\zeta}\right) \frac{a|\nabla g|^{4}}{(\xi-g)^{4}} .
\end{gathered}
$$

Now we claim that

$$
\Xi \geq 0
$$

Indeed, recalling (2.4), (2.26) and (2.31),

$$
\Xi=2 g^{\prime}-\frac{\sqrt{n}\left|g^{\prime \prime}\right|(\xi-g)}{g^{\prime}}=2 F^{\prime}(u)-\frac{\sqrt{n}\left|F^{\prime \prime}(u)\right| u(\xi-G(u))}{F^{\prime}(u)}
$$

and therefore (2.33) is a consequence of (1.17).

We also remark that $\nabla|\nabla g|^{2}=2 D^{2} g \nabla g$, and consequently

$$
\begin{aligned}
0 & \leq\left(\frac{\left\langle\nabla|\nabla g|^{2}, \nabla g\right\rangle}{2(\xi-g)|\nabla g|^{2}}+\frac{|\nabla g|^{2}}{(\xi-g)^{2}}\right)^{2} \\
& =\left(\frac{\left\langle\nabla|\nabla g|^{2}, \nabla g\right\rangle}{2(\xi-g)|\nabla g|^{2}}\right)^{2}+\frac{\left\langle\nabla|\nabla g|^{2}, \nabla g\right\rangle}{(\xi-g)^{3}}+\frac{|\nabla g|^{4}}{(\xi-g)^{4}} \\
& =\left(\frac{\left\langle D^{2} g \nabla g, \nabla g\right\rangle}{(\xi-g)|\nabla g|^{2}}\right)^{2}+\frac{\left\langle\nabla|\nabla g|^{2}, \nabla g\right\rangle}{(\xi-g)^{3}}+\frac{|\nabla g|^{4}}{(\xi-g)^{4}} \\
& \leq \frac{\left|D^{2} g\right|^{2}}{(\xi-g)^{2}}+\frac{\left\langle\nabla|\nabla g|^{2}, \nabla g\right\rangle}{(\xi-g)^{3}}+\frac{|\nabla g|^{4}}{(\xi-g)^{4}} .
\end{aligned}
$$

This information, (2.32) and (2.33) give that

$$
\left[2 g^{\prime}-\zeta n\left(\frac{g^{\prime \prime}}{g^{\prime}}\right)^{2}(\xi-g)^{2}\right] \frac{a\left|D^{2} g\right|^{2}}{(\xi-g)^{2}}+\frac{2 a g^{\prime}\left\langle\nabla|\nabla g|^{2}, \nabla g\right\rangle}{(\xi-g)^{3}}+\left(2 g^{\prime}-\frac{1}{\zeta}\right) \frac{a|\nabla g|^{4}}{(\xi-g)^{4}}
$$




$$
\geq \zeta n\left(\frac{g^{\prime \prime}}{g^{\prime}}\right)^{2} \frac{a\left\langle\nabla|\nabla g|^{2}, \nabla g\right\rangle}{\xi-g}+\left(\zeta n\left(\frac{g^{\prime \prime}}{g^{\prime}}\right)^{2}(\xi-g)^{2}-\frac{1}{\zeta}\right) \frac{a|\nabla g|^{4}}{(\xi-g)^{4}} .
$$

Now, we insert this inequality into (2.29), thus finding that

$$
\begin{aligned}
a g^{\prime} \Delta w-w_{t} \geq \zeta n & \left(\frac{g^{\prime \prime}}{g^{\prime}}\right)^{2} \frac{a\left\langle\nabla|\nabla g|^{2}, \nabla g\right\rangle}{\xi-g}+\left(2(\xi-g)+\zeta n\left(\frac{g^{\prime \prime}}{g^{\prime}}\right)^{2}(\xi-g)^{2}-\frac{1}{\zeta}\right) \frac{a|\nabla g|^{4}}{(\xi-g)^{4}} \\
+ & 2 a\left(\frac{g^{\prime}}{\xi-g}-1\right)\langle\nabla w, \nabla g\rangle-\frac{2 \mu_{1}|\nabla g|^{2}}{(\xi-g)^{2}}-\frac{2 \gamma_{1}|\nabla g|}{(\xi-g)^{2}}-\Upsilon_{3} .
\end{aligned}
$$

Moreover, it is convenient to exploit (2.16) once again and note that

$$
\frac{\left\langle\nabla|\nabla g|^{2}, \nabla g\right\rangle}{(\xi-g)^{2}}+\frac{2|\nabla g|^{4}}{(\xi-g)^{3}}=\left\langle\frac{\nabla|\nabla g|^{2}}{(\xi-g)^{2}}+2 \frac{|\nabla g|^{2} \nabla g}{(\xi-g)^{3}}, \nabla g\right\rangle=\langle\nabla w, \nabla g\rangle
$$

and consequently

$$
\begin{aligned}
& \zeta n\left(\frac{g^{\prime \prime}}{g^{\prime}}\right)^{2} \frac{a\left\langle\nabla|\nabla g|^{2}, \nabla g\right\rangle}{\xi-g}+\left(2(\xi-g)+\zeta n\left(\frac{g^{\prime \prime}}{g^{\prime}}\right)^{2}(\xi-g)^{2}-\frac{1}{\zeta}\right) \frac{a|\nabla g|^{4}}{(\xi-g)^{4}} \\
=\zeta n a\left(\frac{g^{\prime \prime}}{g^{\prime}}\right)^{2}(\xi-g)\left(\langle\nabla w, \nabla g\rangle-\frac{2|\nabla g|^{4}}{(\xi-g)^{3}}\right) & +\left(2(\xi-g)+\zeta n\left(\frac{g^{\prime \prime}}{g^{\prime}}\right)^{2}(\xi-g)^{2}-\frac{1}{\zeta}\right) \frac{a|\nabla g|^{4}}{(\xi-g)^{4}} \\
& +\left(2(\xi-g)-\zeta n\left(\frac{g^{\prime \prime}}{g^{\prime}}\right)^{2}(\xi-g)^{2}-\frac{1}{\zeta}\right) \frac{a|\nabla g|^{4}}{(\xi-g)^{4}} .
\end{aligned}
$$

We can thereby plug this information into (2.34) and deduce that

$$
\begin{aligned}
a g^{\prime} \Delta w-w_{t} \geq & \left(2(\xi-g)-\zeta n\left(\frac{g^{\prime \prime}}{g^{\prime}}\right)^{2}(\xi-g)^{2}-\frac{1}{\zeta}\right) \frac{a|\nabla g|^{4}}{(\xi-g)^{4}} \\
& +a\left(\frac{2 g^{\prime}}{\xi-g}-2+\zeta n\left(\frac{g^{\prime \prime}}{g^{\prime}}\right)^{2}(\xi-g)\right)\langle\nabla w, \nabla g\rangle \\
& -\frac{2 \mu_{1}|\nabla g|^{2}}{(\xi-g)^{2}}-\frac{2 \gamma_{1}|\nabla g|}{(\xi-g)^{2}}-\Upsilon_{3} .
\end{aligned}
$$

Now we remark that

$$
\begin{aligned}
2(\xi- & g)-\zeta n\left(\frac{g^{\prime \prime}}{g^{\prime}}\right)^{2}(\xi-g)^{2}-\frac{1}{\zeta}=2(\xi-g)\left(1-\sqrt{n} \frac{\left|g^{\prime \prime}\right|}{g^{\prime}}\right) \\
= & 2(\xi-g)\left(1-\sqrt{n} \frac{\left|F^{\prime \prime}(u)\right| u}{F^{\prime}(u)}\right) \geq 2 \kappa(\xi-g),
\end{aligned}
$$

thanks to (1.12), (2.4), (2.7) and (2.26). 
For this reason, recalling the definition of $w$ in (2.6), we obtain that

$$
\left(2(\xi-g)-\zeta n\left(\frac{g^{\prime \prime}}{g^{\prime}}\right)^{2}(\xi-g)^{2}-\frac{1}{\zeta}\right) \frac{a|\nabla g|^{4}}{(\xi-g)^{4}} \geq \frac{2 \kappa a|\nabla g|^{4}}{(\xi-g)^{3}}=2 a \kappa(\xi-g) w^{2}
$$

Hence, by (2.35),

$$
\begin{gathered}
a g^{\prime} \Delta w-w_{t} \geq 2 a \kappa(\xi-g) w^{2}+a\left(\frac{2 g^{\prime}}{\xi-g}-2+\zeta n\left(\frac{g^{\prime \prime}}{g^{\prime}}\right)^{2}(\xi-g)\right)\langle\nabla w, \nabla g\rangle \\
-\frac{2 \mu_{1}|\nabla g|^{2}}{(\xi-g)^{2}}-\frac{2 \gamma_{1}|\nabla g|}{(\xi-g)^{2}}-\Upsilon_{3}
\end{gathered}
$$

We also note that

$$
\frac{2 g^{\prime}}{\xi-g}-2+\zeta n\left(\frac{g^{\prime \prime}}{g^{\prime}}\right)^{2}(\xi-g)=\frac{2 g^{\prime}}{\xi-g}-2+\sqrt{n} \frac{\left|g^{\prime \prime}\right|}{g^{\prime}}=2 \lambda
$$

thanks to (2.2) and (2.26).

Using this identity and (2.6) inside (2.36), we get that

$$
a g^{\prime} \Delta w-w_{t} \geq 2 a \kappa(\xi-g) w^{2}+2 a \lambda\langle\nabla w, \nabla g\rangle-2 \mu_{1} w-\frac{2 \gamma_{1}|\nabla g|}{(\xi-g)^{2}}-\Upsilon_{3}
$$

Now we observe that, by (2.22), (2.23) and (2.30),

$$
\begin{gathered}
\Upsilon_{3}=\frac{2\left\langle\nabla g, \Upsilon_{1}\right\rangle}{(\xi-g)^{2}}+\frac{2 g^{\prime} \Delta g\left\langle\nabla g, \nabla a+\partial_{u} a \nabla u\right\rangle}{(\xi-g)^{2}}+\frac{2\left\langle\nabla g, \nabla a+\partial_{u} a \nabla u\right\rangle|\nabla g|^{2}}{(\xi-g)^{2}} \\
=\frac{2}{(\xi-g)^{2}}\left\langle\nabla g, \frac{g^{\prime}(v)}{u}\left(\sum_{i=1}^{n} \partial_{\omega_{i}} H \nabla \partial_{x_{i}} u+\sum_{i, j=1}^{n} \partial_{\Omega_{i j}} H \nabla \partial_{x_{i} x_{j}}^{2} u\right)\right\rangle \\
+\frac{2 g^{\prime} \Delta g\left\langle\nabla g, \nabla a+\partial_{u} a \nabla u\right\rangle}{(\xi-g)^{2}}+\frac{2\left\langle\nabla g, \nabla a+\partial_{u} a \nabla u\right\rangle|\nabla g|^{2}}{(\xi-g)^{2}} .
\end{gathered}
$$

From (2.8) and (2.12), we also note that

$$
\Delta g=g^{\prime \prime}|\nabla v|^{2}+g^{\prime} \Delta v
$$

and

$$
\Delta v=\operatorname{div}\left(\frac{\nabla u}{u}\right)=\frac{\Delta u}{u}-\frac{|\nabla u|^{2}}{u^{2}}=\frac{\Delta u}{u}-|\nabla v|^{2} .
$$


These observations lead to

$$
\begin{aligned}
g^{\prime} \Delta g & +|\nabla g|^{2}=g^{\prime} g^{\prime \prime}|\nabla v|^{2}+\left(g^{\prime}\right)^{2} \Delta v+\left(g^{\prime}\right)^{2}|\nabla v|^{2} \\
& =g^{\prime} g^{\prime \prime}|\nabla v|^{2}+\left(g^{\prime}\right)^{2}\left(\frac{\Delta u}{u}-|\nabla v|^{2}\right)+\left(g^{\prime}\right)^{2}|\nabla v|^{2} \\
& =F^{\prime}(u)\left(u F^{\prime \prime}(u) \frac{|\nabla u|^{2}}{u^{2}}+F^{\prime}(u) \frac{\Delta u}{u}\right) \\
& =\frac{F^{\prime}(u)\left(\operatorname{div}\left(F^{\prime}(u) \nabla u\right)\right)}{u} .
\end{aligned}
$$

As a result, after an interesting cancellation we conclude that

$$
\begin{aligned}
& \frac{2 g^{\prime} \Delta g\left\langle\nabla g, \partial_{u} a \nabla u\right\rangle}{(\xi-g)^{2}}+\frac{2\left\langle\nabla g, \partial_{u} a \nabla u\right\rangle|\nabla g|^{2}}{(\xi-g)^{2}} \\
& =\frac{2 F^{\prime}(u)\left(\operatorname{div}\left(F^{\prime}(u) \nabla u\right)\right) \partial_{u} a\langle\nabla g, \nabla u\rangle}{u(\xi-g)^{2}}=\frac{2\left(\operatorname{div}\left(F^{\prime}(u) \nabla u\right)\right) \partial_{u} a\langle\nabla g, \nabla g\rangle}{(\xi-g)^{2}} \\
& =2 \partial_{u} a\left(\operatorname{div}\left(F^{\prime}(u) \nabla u\right)\right) w \leq 2 \mu_{2} w,
\end{aligned}
$$

where the definition of $\mu_{2}$ given in (1.20) has been used in the inequality. Using again (2.39),

$$
\begin{aligned}
& \frac{2 g^{\prime} \Delta g\langle\nabla g, \nabla a\rangle}{(\xi-g)^{2}}+\frac{2\langle\nabla g, \nabla a\rangle|\nabla g|^{2}}{(\xi-g)^{2}} \\
& \quad=\frac{2 F^{\prime}(u)\left(\operatorname{div}\left(F^{\prime}(u) \nabla u\right)\right)\langle\nabla g, \nabla a\rangle}{u(\xi-g)^{2}} \leq \frac{2 \gamma_{2}|\nabla g|}{(\xi-g)^{2}},
\end{aligned}
$$

where we have used the definition of $\gamma_{2}$ in (1.22).

From (2.38), (2.40) and (2.41), we see that

$$
\Upsilon_{3} \leq \frac{2}{(\xi-g)^{2}}\left\langle\nabla g, \frac{g^{\prime}(v)}{u}\left(\sum_{i=1}^{n} \partial_{\omega_{i}} H \nabla \partial_{x_{i}} u+\sum_{i, j=1}^{n} \partial_{\Omega_{i j}} H \nabla \partial_{x_{i} x_{j}}^{2} u\right)\right\rangle+2 \mu_{2} w+\frac{2 \gamma_{2}|\nabla g|}{(\xi-g)^{2}}
$$

Now, recalling the definition of $\gamma_{3}$ in (1.23), we have that

$$
\begin{aligned}
& \left|\frac{g^{\prime}(v)}{u}\left(\sum_{i=1}^{n} \partial_{\omega_{i}} H \nabla \partial_{x_{i}} u+\sum_{i, j=1}^{n} \partial_{\Omega_{i j}} H \nabla \partial_{x_{i} x_{j}}^{2} u\right)\right| \\
& \quad=\left|\frac{F^{\prime}(u)}{u}\left(\sum_{i=1}^{n} \partial_{\omega_{i}} H \nabla \partial_{x_{i}} u+\sum_{i, j=1}^{n} \partial_{\Omega_{i j}} H \nabla \partial_{x_{i} x_{j}}^{2} u\right)\right| \leq \gamma_{3} .
\end{aligned}
$$

This and (2.42) yield that

$$
\Upsilon_{3} \leq \frac{2\left(\gamma_{2}+\gamma_{3}\right)|\nabla g|}{(\xi-g)^{2}}+2 \mu_{2} w
$$

Combining this estimate and (2.37) we obtain the desired result in (2.9). 


\section{Cut-off FunCtions AND LOCALizAtion PROCEDURES}

In order to obtain the global bounds in Theorem 1.5, we distinguish four regimes, according to the cut-off functions in (1.27). For this, we recall the following auxiliary cut-off functions, both in the space and in the time variables, that have been introduced in Lemmata 2.2 and 2.3 in [10]:

Lemma 3.1. Let $\theta \in(0,1), R>0$ and $\rho \in(0, R)$. Then, there exists a decreasing function $\bar{\psi} \in C^{2}(\mathbb{R},[0,1])$ such that

$$
\bar{\psi}(r)=1 \text { for all } r \in[0, R-\rho], \quad \bar{\psi}(r)=0 \text { for all } r \geq R,
$$

and, for every $r \geq 0$,

$$
\rho\left|\bar{\psi}^{\prime}(r)\right|+\rho^{2}\left|\bar{\psi}^{\prime \prime}(r)\right| \leq C(\bar{\psi}(r))^{\theta}
$$

for some $C>0$, depending only on $\theta$.

Lemma 3.2. Let $t_{0} \in \mathbb{R}$ and $T>0$. Let $\theta \in(0,1)$ and $\delta \in(0, T)$. Then, there exists an increasing function $\phi \in$ $C^{2}(\mathbb{R},[0,1])$ such that

$$
\phi(t)=0 \text { for all } t \leq t_{0}-T \text {, and } \phi(t)=1 \text { for all } t \geq t_{0}-T+\delta,
$$

and, for every $t \in \mathbb{R}$,

$$
\delta\left|\phi^{\prime}(t)\right| \leq C(\phi(t))^{\frac{1+\theta}{2}}
$$

for some $C>0$, depending only on $\theta$.

Now we obtain a general inequality for the auxiliary barrier $w$ introduced in (2.6) in dependence of a smooth and positive function $\psi$ :

Lemma 3.3. Let $\psi \in C^{\infty}\left(Q_{R, T},(0,+\infty)\right)$. Then, there exists $C>0$, depending only on $a_{0}$ and $\kappa$, such that

$$
\begin{aligned}
& \frac{a g^{\prime} \Delta(w \psi)-(w \psi)_{t}}{2}-a\left\langle\nabla(w \psi), \frac{g^{\prime} \nabla \psi}{\psi}+\lambda \nabla g\right\rangle \\
\geq & \frac{a_{0} \kappa(\xi-g) w^{2} \psi}{4}-a \lambda w\langle\nabla \psi, \nabla g\rangle-\frac{C \mu^{2} \psi}{(\xi-g)}-\frac{C \gamma^{4 / 3} \psi}{(\xi-g)^{5 / 3}} \\
& -\frac{a g^{\prime} w|\nabla \psi|^{2}}{\psi}+\frac{\left(a g^{\prime} \Delta \psi-\psi_{t}\right) w}{2} .
\end{aligned}
$$

Proof. We have that

$$
\begin{aligned}
& \frac{a g^{\prime} \Delta(w \psi)-(w \psi)_{t}}{2}-\frac{a g^{\prime}\langle\nabla(w \psi), \nabla \psi\rangle}{\psi} \\
& =\frac{\left(a g^{\prime} \Delta w-w_{t}\right) \psi}{2}+\frac{\left(a g^{\prime} \Delta \psi-\psi_{t}\right) w}{2}-\frac{a g^{\prime} w|\nabla \psi|^{2}}{\psi}
\end{aligned}
$$


Hence, subtracting $a \lambda\langle\nabla(w \psi), \nabla g\rangle$ to both sides of (3.6),

$$
\begin{aligned}
& \frac{a g^{\prime} \Delta(w \psi)-(w \psi)_{t}}{2}-a\left\langle\nabla(w \psi), \frac{g^{\prime} \nabla \psi}{\psi}+\lambda \nabla g\right\rangle \\
& =\frac{\left(a g^{\prime} \Delta w-w_{t}\right) \psi}{2}+\frac{\left(a g^{\prime} \Delta \psi-\psi_{t}\right) w}{2}-\frac{a g^{\prime} w|\nabla \psi|^{2}}{\psi}-a \lambda\langle\nabla(w \psi), \nabla g\rangle .
\end{aligned}
$$

As a result, it follows from Lemma 2.1 that

$$
\begin{gathered}
\frac{a g^{\prime} \Delta(w \psi)-(w \psi)_{t}}{2}-a\left\langle\nabla(w \psi), \frac{g^{\prime} \nabla \psi}{\psi}+\lambda \nabla g\right\rangle \\
\geq a \kappa(\xi-g) w^{2} \psi+a \lambda\langle\nabla w, \nabla g\rangle \psi-\mu w \psi-\frac{\gamma|\nabla g| \psi}{(\xi-g)^{2}} \\
-a \lambda\langle\nabla(w \psi), \nabla g\rangle-\frac{a g^{\prime} w|\nabla \psi|^{2}}{\psi}+\frac{\left(a g^{\prime} \Delta \psi-\psi_{t}\right) w}{2} .
\end{gathered}
$$

One can also notice that

$$
\langle\nabla w, \nabla g\rangle \psi-\langle\nabla(w \psi), \nabla g\rangle=-w\langle\nabla \psi, \nabla g\rangle
$$

which together with (1.10) and (3.7) implies that

$$
\begin{aligned}
& \frac{a g^{\prime} \Delta(w \psi)-(w \psi)_{t}}{2}-a\left\langle\nabla(w \psi), \frac{g^{\prime} \nabla \psi}{\psi}+\lambda \nabla g\right\rangle \\
\geq & a_{0} \kappa(\xi-g) w^{2} \psi-a \lambda w\langle\nabla \psi, \nabla g\rangle-\mu w \psi-\frac{\gamma|\nabla g| \psi}{(\xi-g)^{2}} \\
& -\frac{a g^{\prime} w|\nabla \psi|^{2}}{\psi}+\frac{\left(a g^{\prime} \Delta \psi-\psi_{t}\right) w}{2} .
\end{aligned}
$$

In addition, from (2.6) and Young's inequality with exponents 4 and 4/3,

$$
\begin{gathered}
\frac{\gamma|\nabla g| \psi}{(\xi-g)^{2}}=\frac{\gamma \psi \sqrt{w}}{\xi-g}=\sqrt[4]{\xi-g} \sqrt{w} \sqrt[4]{\psi} \frac{\gamma \psi^{\frac{3}{4}}}{(\xi-g)^{\frac{5}{4}}} \\
\leq \frac{a_{0} \kappa(\xi-g) w^{2} \psi}{2}+\frac{C \gamma^{4 / 3} \psi}{\left(a_{0} \kappa\right)^{1 / 3}(\xi-g)^{5 / 3}} \\
\leq \frac{a_{0} \kappa(\xi-g) w^{2} \psi}{2}+\frac{C \gamma^{4 / 3} \psi}{(\xi-g)^{5 / 3}}
\end{gathered}
$$

for some $C>0$, possibly varying from line to line and possibly depending only on $a_{0}$ and $\kappa$. Formulas (3.8) and (3.9) entail that

$$
\begin{aligned}
& \frac{a g^{\prime} \Delta(w \psi)-(w \psi)_{t}}{2}-a\left\langle\nabla(w \psi), \frac{g^{\prime} \nabla \psi}{\psi}+\lambda \nabla g\right\rangle \\
\geq & \frac{a_{0} \kappa(\xi-g) w^{2} \psi}{2}-a \lambda w\langle\nabla \psi, \nabla g\rangle-\mu w \psi-\frac{C \gamma^{4 / 3} \psi}{(\xi-g)^{5 / 3}} \\
& -\frac{a g^{\prime} w|\nabla \psi|^{2}}{\psi}+\frac{\left(a g^{\prime} \Delta \psi-\psi_{t}\right) w}{2} .
\end{aligned}
$$


Besides, by the Cauchy-Schwarz inequality,

$$
\begin{gathered}
\mu w \psi=(\sqrt{\xi-g} w \sqrt{\psi})\left(\frac{\mu \sqrt{\psi}}{\sqrt{\xi-g}}\right) \leq \frac{a_{0} \kappa(\xi-g) w^{2} \psi}{4}+\frac{C \mu^{2} \psi}{a_{0} \kappa(\xi-g)} \\
\leq \frac{a_{0} \kappa(\xi-g) w^{2} \psi}{4}+\frac{C \mu^{2} \psi}{(\xi-g)}
\end{gathered}
$$

which combined with (3.10) gives the desired result in (3.5).

Inequality (3.5) will play a pivotal a role in the following computations in order to analyze four different regimes, as given by (1.27).

Lemma 3.4. In the setting of Theorem 1.5, if $x \in B\left(x_{0}, R-\rho\right)$ and $t \in\left[t_{0}-T, t_{0}\right]$,

$$
w \leq\left[\tau_{u}^{2}+C\left(\mu+\gamma^{2 / 3}+\frac{1}{\rho^{2}}+\frac{1}{\rho(R-\rho)}+\frac{\sqrt{k_{+}}}{\rho}\right)\right],
$$

for some $C>0$, depending only on $n, \eta, a_{0}, \kappa$ and $\Gamma$. Here, $\tau_{u}, \mu$ and $\gamma$ are the quantities defined in (1.26), (1.21) and (1.24), respectively.

Proof. Let $\theta \in(0,1)$, to be conveniently chosen in what follows. For every $x \in B\left(x_{0}, R\right)$, we define

$$
\psi(x):=\bar{\psi}\left(\mathrm{d}\left(x, x_{0}\right)\right)
$$

where $\mathrm{d}(\cdot, \cdot)$ represents the geodesic distance and $\bar{\psi}$ is the function introduced in Lemma 3.1.

Recalling the assumption (1.9) on the Ricci curvature, we have that

$$
\Delta \mathrm{d}\left(x, x_{0}\right) \leq \frac{n-1}{\mathrm{~d}\left(x, x_{0}\right)}+\sqrt{(n-1) k_{+}} .
$$

As a result, in view of (3.2), we deduce that

$$
\text { and } \begin{aligned}
|\nabla \psi(x)|= & \left|\bar{\psi}^{\prime}\left(\mathrm{d}\left(x, x_{0}\right)\right) \nabla \mathrm{d}\left(x, x_{0}\right)\right| \leq \frac{C(\psi(x))^{\theta}}{\rho} \\
-\Delta \psi(x) & =-\bar{\psi}^{\prime}\left(\mathrm{d}\left(x, x_{0}\right)\right) \Delta \mathrm{d}\left(x, x_{0}\right)-\bar{\psi}^{\prime \prime}\left(\mathrm{d}\left(x, x_{0}\right)\right)\left|\nabla \mathrm{d}\left(x, x_{0}\right)\right| \\
\leq & \frac{C(\psi(x))^{\theta}}{\rho}\left(\frac{n-1}{\mathrm{~d}\left(x, x_{0}\right)}+\sqrt{(n-1) k_{+}}\right)+\frac{C(\psi(x))^{\theta}}{\rho^{2}},
\end{aligned}
$$

with $C>0$ depending only on $\theta$.

We now define $\widetilde{w}:=w \psi$ and, in the support of $\psi$, we exploit (3.5) and write that

$$
\begin{aligned}
& \frac{a g^{\prime} \Delta \widetilde{w}-\widetilde{w}_{t}}{2}-a\left\langle\nabla \widetilde{w}, \frac{g^{\prime} \nabla \psi}{\psi}+\lambda \nabla g\right\rangle \\
\geq & \frac{a_{0} \kappa(\xi-g) w^{2} \psi}{4}-a \lambda w\langle\nabla \psi, \nabla g\rangle-\frac{C \mu^{2} \psi}{(\xi-g)}-\frac{C \gamma^{4 / 3} \psi}{(\xi-g)^{5 / 3}} \\
& -\frac{a g^{\prime} w|\nabla \psi|^{2}}{\psi}+\frac{a g^{\prime} w \Delta \psi}{2} .
\end{aligned}
$$


We take $\left(x_{1}, t_{1}\right)$ in the closure of $Q_{R, T}$ such that

$$
\sup _{Q_{R, T}} \widetilde{w}=\widetilde{w}\left(x_{1}, t_{1}\right)
$$

Since $\widetilde{w}(x, t)=0$ if $x \in \partial B\left(x_{0}, R\right)$, necessarily $x_{1}$ is an interior point of $B\left(x_{0}, R\right)$. Consequently $\nabla \widetilde{w}\left(x_{1}, t_{1}\right)=0$ and $\Delta \widetilde{w}\left(x_{1}, t_{1}\right) \leq 0$. Hence, inserting this information into (3.15), we obtain that

$$
\begin{aligned}
0 \geq & \frac{\widetilde{w}_{t}}{2}+\frac{a_{0} \kappa(\xi-g) w^{2} \psi}{4}-a \lambda w\langle\nabla \psi, \nabla g\rangle-\frac{C \mu^{2} \psi}{(\xi-g)}-\frac{C \gamma^{4 / 3} \psi}{(\xi-g)^{5 / 3}} \\
& -\frac{a g^{\prime} w|\nabla \psi|^{2}}{\psi}+\left.\frac{a g^{\prime} w \Delta \psi}{2}\right|_{(x, t)=\left(x_{1}, t_{1}\right)} .
\end{aligned}
$$

Exploiting (2.1), (2.4), (2.5) and (2.6), we also see that

$$
\begin{gathered}
\frac{\left|F^{\prime}(u(x, t))\right|^{2}|\nabla u(x, t)|^{2}}{|u(x, t)|^{2}(\xi-G(u(x, t)))^{2}}=\frac{\left|g^{\prime}(v(x, t))\right|^{2}|\nabla u(x, t)|^{2}}{|u(x, t)|^{2}(\xi-g(v(x, t)))^{2}} \\
=\frac{\left|g^{\prime}(v(x, t))\right|^{2}|\nabla v(x, t)|^{2}}{(\xi-g(v(x, t)))^{2}}=w(x, t) .
\end{gathered}
$$

Now, to address the proof of (3.12), it is convenient to distinguish two cases, namely:

$$
\begin{aligned}
& \text { either } t_{1}=t_{0}-T \text {, } \\
& \text { or } t_{1} \in\left(t_{0}-T, t_{0}\right] \text {. }
\end{aligned}
$$

To start with, we suppose that (3.19) holds true. In this case, we use (1.26), (3.16) and (3.18) to deduce that, for every $(x, t) \in Q_{R, T}$,

$$
\begin{aligned}
\widetilde{w}(x, t) & \leq \widetilde{w}\left(x_{1}, t_{0}-T\right) \\
& \leq \sup _{x \in B\left(x_{0}, R\right)} \widetilde{w}\left(x, t_{0}-T\right) \\
& \leq \sup _{x \in B\left(x_{0}, R\right)} w\left(x, t_{0}-T\right) \\
& =\sup _{x \in B\left(x_{0}, R\right)} \frac{\left(F^{\prime}(u)\right)^{2}|\nabla u|^{2}}{u^{2}(\xi-G(u))^{2}}\left(x, t_{0}-T\right) \\
& \leq \tau_{u}^{2} .
\end{aligned}
$$

In particular, for all $(x, t) \in B\left(x_{0}, R-\rho\right) \times\left[t_{0}-T, t_{0}\right]$,

$$
w(x, t)=\widetilde{w}(x, t) \leq \tau_{u}^{2},
$$

and this proves (3.12) in this case. 
Hence, to complete the proof of (3.12), we now consider the case in which (3.20) is satisfied. Then, $\widetilde{w}_{t}\left(x_{1}, t_{1}\right) \geq$ 0 , and consequently (3.17) entails that

$$
\begin{aligned}
0 \geq & \frac{a_{0} \kappa(\xi-g) w^{2} \psi}{4}-a \lambda w\langle\nabla \psi, \nabla g\rangle-\frac{C \mu^{2} \psi}{(\xi-g)}-\frac{C \gamma^{4 / 3} \psi}{(\xi-g)^{5 / 3}} \\
& -\frac{a g^{\prime} w|\nabla \psi|^{2}}{\psi}+\left.\frac{a g^{\prime} w \Delta \psi}{2}\right|_{(x, t)=\left(x_{1}, t_{1}\right)}
\end{aligned}
$$

It is also useful to observe that

$$
\frac{\sqrt{n}\left|g^{\prime \prime}(r)\right|}{g^{\prime}(r)}=\frac{\sqrt{n} e^{r}\left|F^{\prime \prime}\left(e^{r}\right)\right|}{F^{\prime}\left(e^{r}\right)} \leq \frac{2 F^{\prime}\left(e^{r}\right)}{\xi-G\left(e^{r}\right)}=\frac{2 g^{\prime}(r)}{\xi-g(r)}
$$

thanks to (2.4) and (1.17).

From this and the definition of $\lambda$ in (2.2), we conclude that

$$
|\lambda(r)| \leq \frac{2 g^{\prime}(r)}{\xi-g(r)}+1
$$

It is also useful to observe that, in light of (1.16), (2.1) and (2.4),

$$
\frac{2 g^{\prime}(r)}{\xi-g(r)}=\frac{2 F^{\prime}\left(e^{r}\right)}{\xi-G\left(e^{r}\right)} \leq 2 \Gamma
$$

and thus $|\lambda(r)| \leq 2 \Gamma+1$.

As a consequence, recalling the definition of $w$ in (2.6), and utilizing the Young's inequality with exponents $4 / 3$ and 4 , we see that, in the support of $\psi$,

$$
\begin{aligned}
a \mid \lambda w & \langle\nabla \psi, \nabla g\rangle\left|\leq(2 \Gamma+1) a_{0}^{-1} w\right| \nabla \psi|| \nabla g \mid \\
& =(2 \Gamma+1) a_{0}^{-1} w^{3 / 2}|\nabla \psi|(\xi-g) \\
& =(2 \Gamma+1)\left(\frac{a_{0}^{3 / 4}[\kappa(\xi-g)]^{3 / 4} w^{3 / 2} \psi^{3 / 4}}{3}\right) \frac{3 a_{0}^{-7 / 4}(\xi-g)^{1 / 4}|\nabla \psi|}{\kappa^{3 / 4} \psi^{3 / 4}} \\
& \leq \frac{1}{8} a_{0} \kappa(\xi-g) w^{2} \psi+\frac{C(\xi-g)|\nabla \psi|^{4}}{\psi^{3}}
\end{aligned}
$$

for some constant $C>0$ depending only on $a_{0}, \kappa$ and $\Gamma$. From this and (3.14), we find that

$$
a|\lambda w\langle\nabla \psi, \nabla g\rangle| \leq \frac{1}{8} a_{0}(\xi-g) \kappa w^{2} \psi+\frac{C(\xi-g) \psi^{4 \theta-3}}{\rho^{4}},
$$

up to renaming $C>0$, possibly also in dependence of $\theta$. 
Also, by using again (2.4), (3.14), (3.22) and the Cauchy-Schwarz inequality, we can write that

$$
\begin{aligned}
\frac{a g^{\prime} w|\nabla \psi|^{2}}{\psi} \leq & \frac{C a_{0}^{-1} g^{\prime} w \psi^{2 \theta-1}}{\rho^{2}}=a_{0}^{-1} g^{\prime} \sqrt{\xi-g} w \sqrt{\psi} \frac{C \psi^{2 \theta-\frac{3}{2}}}{\sqrt{\xi-g} \rho^{2}} \\
& \leq \frac{1}{16} a_{0}(\xi-g) \kappa w^{2} \psi+\frac{C\left(g^{\prime}\right)^{2} \psi^{4 \theta-3}}{a_{0}^{3}(\xi-g) \kappa \rho^{4}} \\
& \leq \frac{1}{16} a_{0}(\xi-g) \kappa w^{2} \psi+\frac{C(\xi-g) \psi^{4 \theta-3}}{\rho^{4}}
\end{aligned}
$$

up to renaming $C$.

Plugging this information and (3.23) into (3.21), we obtain that, at the point $\left(x_{1}, t_{1}\right)$,

$$
\frac{a_{0} \kappa(\xi-g) w^{2} \psi}{16} \leq \frac{C \mu^{2} \psi}{(\xi-g)}+\frac{C \gamma^{4 / 3} \psi}{(\xi-g)^{5 / 3}}+\frac{C(\xi-g) \psi^{4 \theta-3}}{\rho^{4}}-\frac{a g^{\prime} w \Delta \psi}{2} .
$$

Moreover, by (3.14), using the short notation $d:=\mathrm{d}\left(x, x_{0}\right)$, we see that

$$
\begin{aligned}
-\frac{\Delta \psi w}{2} & \leq \frac{C w}{2}\left[\frac{\psi^{\theta}}{\rho}\left(\frac{n-1}{d}+\sqrt{(n-1) k_{+}}\right)+\frac{\psi^{\theta}}{\rho^{2}}\right] \\
& =\frac{C \sqrt{\xi-g} w \sqrt{\psi}}{2}\left[\rho\left(\frac{n-1}{d}+\sqrt{(n-1) k_{+}}\right)+1\right] \frac{\psi^{\theta-\frac{1}{2}}}{\sqrt{\xi-g} \rho^{2}} .
\end{aligned}
$$

Consequently, by the Cauchy-Schwarz inequality and (3.22),

$$
\begin{aligned}
-\frac{a g^{\prime} \Delta \psi w}{2} & \leq \frac{a_{0} \kappa(\xi-g) w^{2} \psi}{32}+C\left[\rho\left(\frac{n-1}{d}+\sqrt{(n-1) k_{+}}\right)+1\right]^{2} \frac{a_{0}^{-3}\left(g^{\prime}\right)^{2} \psi^{2 \theta-1}}{(\xi-g) \kappa \rho^{4}} \\
& \leq \frac{a_{0} \kappa(\xi-g) w^{2} \psi}{32}+C\left[\rho\left(\frac{n-1}{d}+\sqrt{(n-1) k_{+}}\right)+1\right]^{2} \frac{\psi^{2 \theta-1}(\xi-g)}{\rho^{4}}
\end{aligned},
$$

up to renaming $C$.

We also remark that when $x \in B\left(x_{0}, R-\rho\right)$, we have that $d=\mathrm{d}\left(x, x_{0}\right) \in[0, R-\rho)$, and thus $\frac{R-d}{\rho}>1$, which gives that $\psi(x)=\bar{\psi}(d)=\alpha\left(\frac{R-d}{\rho}\right)=1$. In particular,

$$
\Delta \psi(x)=0 \quad \text { for all } x \in B\left(x_{0}, R-\rho\right) .
$$

Notice also that

$$
\begin{aligned}
-\frac{a g^{\prime} \Delta \psi w}{2} & \leq \frac{a_{0} \kappa(\xi-g) w^{2} \psi}{32}+C\left[\rho\left(\frac{n-1}{R-\rho}+\sqrt{(n-1) k_{+}}\right)+1\right]^{2} \frac{\psi^{2 \theta-1}(\xi-g)}{\rho^{4}} \\
& \leq \frac{a_{0} \kappa(\xi-g) w^{2} \psi}{32}+\frac{C \psi^{2 \theta-1}(\xi-g)}{\rho^{2}(R-\rho)^{2}}+\frac{C k_{+} \psi^{2 \theta-1}(\xi-g)}{\rho^{2}}+\frac{C \psi^{2 \theta-1}(\xi-g)}{\rho^{4}}
\end{aligned}
$$

up to renaming $C$. Indeed, the estimate in (3.28) is obvious in $B\left(x_{0}, R-\rho\right)$, since the right hand side vanishes, thanks to (3.27), and it follows directly from (3.26) in the complement of $B\left(x_{0}, R-\rho\right)$, where $d \geq R-\rho$. 
We can therefore insert (3.28) into (3.25) and find that, at the point $\left(x_{1}, t_{1}\right)$,

$$
\begin{aligned}
& \frac{a_{0} \kappa(\xi-g) w^{2} \psi}{32} \leq \frac{C \mu^{2} \psi}{(\xi-g)}+\frac{C \gamma^{4 / 3} \psi}{(\xi-g)^{5 / 3}}+\frac{C \psi^{4 \theta-3}(\xi-g)}{\rho^{4}} \\
& +\frac{C \psi^{2 \theta-1}(\xi-g)}{\rho^{2}(R-\rho)^{2}}+\frac{C k_{+} \psi^{2 \theta-1}(\xi-g)}{\rho^{2}}+\frac{C \psi^{2 \theta-1}(\xi-g)}{\rho^{4}}
\end{aligned}
$$

In light of (3.16), we can rewrite the latter estimate as

$$
\sup _{Q_{R, T}} w^{2} \psi^{2} \leq \frac{C \mu^{2} \psi^{2}}{(\xi-g)^{2}}+\frac{C \gamma^{4 / 3} \psi^{2}}{(\xi-g)^{8 / 3}}+\frac{C \psi^{4 \theta-2}}{\rho^{4}}+\frac{C \psi^{2 \theta}}{\rho^{2}(R-\rho)^{2}}+\frac{C k_{+} \psi^{2 \theta}}{\rho^{2}}+\frac{C \psi^{2 \theta}}{\rho^{4}} .
$$

We recall that $0 \leq \psi \leq 1$ and that $\psi=1$ in $B\left(x_{0}, R-\rho\right)$. In this way, choosing $\theta:=1 / 2$, we deduce from (3.29) and (1.15) that

$$
\begin{gathered}
\sup _{B\left(x_{0}, R-\rho\right) \times\left(t_{0}-T, t_{0}\right]} w^{2}=\sup _{B\left(x_{0}, R-\rho\right) \times\left(t_{0}-T, t_{0}\right]} w^{2} \psi^{2} \\
\leq C \mu^{2}+C \gamma^{4 / 3}+\frac{C}{\rho^{4}}+\frac{C}{\rho^{2}(R-\rho)^{2}}+\frac{C k_{+}}{\rho^{2}},
\end{gathered}
$$

where $C$ can now depend on $\eta$ too. With this, the proof of (3.12) is complete.

Lemma 3.5. In the setting of Theorem 1.5, if $x \in B\left(x_{0}, R\right)$ and $t \in\left[t_{0}-T+\delta, t_{0}\right]$,

$$
w(x, t) \leq\left[\sigma_{u}^{2}+C\left(\mu+\gamma^{2 / 3}+\frac{1}{\delta}\right)\right],
$$

for some $C>0$, depending only on $\eta, a_{0}$ and $\kappa$. Here, $\sigma_{u}, \mu$ and $\gamma$ are the quantities defined in (1.26), (1.21) and (1.24), respectively.

Proof. We take $\phi$ as in Lemma 3.2 (say, with $\theta:=1 / 2$ ), and we define $\widetilde{w}(x, t):=w(x, t) \phi(t)$. Then, in light of $(3.5)$,

$$
\frac{a g^{\prime} \Delta \widetilde{w}-\widetilde{w}_{t}}{2}-a\langle\nabla \widetilde{w}, \lambda \nabla g\rangle \geq \frac{a_{0} \kappa(\xi-g) w^{2} \phi}{4}-\frac{C \mu^{2} \phi}{(\xi-g)}-\frac{C \gamma^{4 / 3} \phi}{(\xi-g)^{5 / 3}}-\frac{\phi_{t} w}{2} .
$$

Suppose that the maximum of $\widetilde{w}$ in the closure of $Q_{R, T}$ is reached at $\left(x_{1}, t_{1}\right)$. Since $\widetilde{w}=0$ when $t=t_{0}-T$, we know that $t_{1} \in\left(t_{0}-T, t_{0}\right]$. As a result,

$$
\widetilde{w}_{t}\left(x_{1}, t_{1}\right) \geq 0
$$

We then distinguish two cases,

$$
\text { either } x_{1} \in \partial B\left(x_{0}, R\right)
$$

$$
\text { or } x_{1} \in B\left(x_{0}, R\right) \text {. }
$$

If (3.33) holds true, then, in $Q_{R, T}$,

$$
\widetilde{w} \leq \widetilde{w}\left(x_{1}, t_{1}\right) \leq \sup _{\substack{x \in \partial B\left(x_{0}, R\right) \\ t \in\left[t_{0}-T, t_{0}\right]}} \widetilde{w}(x, t) \leq \sup _{\substack{x \in \partial B\left(x_{0}, R\right) \\ t \in\left[t_{0}-T, t_{0}\right]}} w(x, t) .
$$


Consequently, recalling the definition of $w$ in (2.6) and using (2.8) and (2.12), we have that

$$
\widetilde{w} \leq \sup _{\substack{x \in \partial B(x 0, R) \\ t \in\left[t_{0}-T+\delta / 2, t_{0}\right]}} \frac{\left(F^{\prime}(u)\right)^{2}|\nabla u|^{2}}{u^{2}(\xi-G(u))^{2}}(x, t)=\sigma_{u}^{2} .
$$

As a result, since $\phi=1$ if $t \geq t_{0}-T+\delta$, thanks to (3.3), we obtain that, if $x \in B\left(x_{0}, R\right)$ and $t \in\left[t_{0}-T+\delta, t_{0}\right]$,

$$
w(x, t)=\widetilde{w}(x, t) \leq \sigma_{u}^{2},
$$

which proves (3.30) in this case.

Hence, we can now suppose that (3.34) holds true. In this case, we have that $\Delta \widetilde{w}\left(x_{1}, t_{1}\right) \leq 0$ and $\nabla \widetilde{w}\left(x_{1}, t_{1}\right)=$ 0 . Therefore, in the light of $(2.4),(3.5)$ and (3.32), at the point $\left(x_{1}, t_{1}\right)$ it holds that

$$
0 \geq \frac{a_{0} \kappa(\xi-g) w^{2} \phi}{4}-\frac{C \mu^{2} \phi}{(\xi-g)}-\frac{C \gamma^{4 / 3} \phi}{(\xi-g)^{5 / 3}}-\frac{\phi_{t} w}{2} .
$$

Moreover, from (3.4) and the Cauchy-Schwarz inequality,

$$
\begin{aligned}
\frac{\phi_{t} w}{2} & \leq \frac{C \phi^{\frac{1+\theta}{2}} w}{2 \delta}=\left(\frac{\sqrt{a_{0} \kappa(\xi-g)} w \sqrt{\phi}}{2}\right)\left(\frac{C \phi^{\frac{\theta}{2}}}{\delta \sqrt{a_{0} \kappa(\xi-g)}}\right) \\
& \leq \frac{a_{0} \kappa(\xi-g) w^{2} \phi}{8}+\frac{C \phi^{\theta}}{\delta^{2} a_{0} \kappa(\xi-g)} \leq \frac{a_{0} \kappa(\xi-g) w^{2} \phi}{8}+\frac{C \phi^{\theta}}{\delta^{2}(\xi-g)} .
\end{aligned}
$$

Plugging (3.36) into (3.35), we conclude that, at the point $\left(x_{1}, t_{1}\right)$,

$$
\frac{1}{8}(\xi-g) a_{0} \kappa w^{2} \phi \leq \frac{C \mu^{2} \phi}{(\xi-g)}+\frac{C \gamma^{4 / 3} \phi}{(\xi-g)^{5 / 3}}+\frac{C \phi^{\theta}}{\delta^{2}(\xi-g)} .
$$

That is, at the point $\left(x_{1}, t_{1}\right)$,

$$
w^{2} \phi \leq \frac{C \mu^{2} \phi}{(\xi-g)^{2}}+\frac{C \gamma^{4 / 3} \phi}{(\xi-g)^{8 / 3}}+\frac{C \phi^{\theta}}{\delta^{2}(\xi-g)^{2}} .
$$

Now, since $0 \leq \phi \leq 1$ and $\phi=1$ for any $t \geq t_{0}-T+\delta$, this implies that

$$
\sup _{B\left(x_{0}, R\right) \times\left[t_{0}-T+\delta, t_{0}\right]} w^{2}=\sup _{B\left(x_{0}, R\right) \times\left[t_{0}-T+\delta, t_{0}\right]} w^{2} \phi^{2} \leq \frac{C \mu^{2}}{(\xi-g)^{2}}+\frac{C}{\delta^{2}(\xi-g)^{2}}+\left.\frac{C \gamma^{4 / 3}}{(\xi-g)^{8 / 3}}\right|_{(x, t)=\left(x_{1}, t_{1}\right)} .
$$

As a consequence, recalling also (1.15), we obtain (3.30), as desired.

Lemma 3.6. In the setting of Theorem 1.5, if $x \in B\left(x_{0}, R\right)$ and $t \in\left[t_{0}-T, t_{0}\right]$,

$$
w(x, t) \leq\left[\sigma_{u}^{2}+\tau_{u}^{2}+C\left(\mu+\gamma^{2 / 3}\right)\right]
$$

for some $C>0$, depending only on $\eta, a_{0}$ and $\kappa$. Here, $\tau_{u}$ and $\sigma_{u}$ are the quantities defined in (1.26), $\mu$ is defined in (1.21), and $\gamma$ is defined in (1.24). 
Proof. We suppose that the maximum of $w$ in the closure of $Q_{R, T}$ is reached at the point $\left(x_{1}, t_{1}\right)$. We distinguish three possibilities:

$$
\begin{aligned}
& \text { either } x_{1} \in B\left(x_{0}, R\right) \text { and } t_{1} \in\left(t_{0}-T, t_{0}\right] \text {, } \\
& \text { or } x_{1} \in B\left(x_{0}, R\right) \text { and } t_{1}=t_{0}-T, \\
& \text { or } x_{1} \in \partial B\left(x_{0}, R\right) \text { and } t_{1} \in\left[t_{0}-T, t_{0}\right] .
\end{aligned}
$$

Suppose first that (3.38) holds true. Then, we have that $\Delta w\left(x_{1}, t_{1}\right) \leq 0, \nabla w\left(x_{1}, t_{1}\right)=0$ and $w_{t}\left(x_{1}, t_{1}\right) \geq 0$. Therefore, in light of Lemma 2.1 and recalling also (2.4), we obtain that, at the point $\left(x_{1}, t_{1}\right)$,

$$
0 \geq a \kappa(\xi-g) w^{2}-\mu w-\frac{\gamma|\nabla g|}{(\xi-g)^{2}} \geq a_{0} \kappa(\xi-g) w^{2}-\mu w-\frac{\gamma|\nabla g|}{(\xi-g)^{2}} .
$$

We insert (3.9) and (3.11) (used here with $\psi:=1)$ into (3.41) to see that, at the point $\left(x_{1}, t_{1}\right)$,

$$
\frac{a_{0} \kappa(\xi-g) w^{2}}{4} \leq \frac{C \mu^{2}}{(\xi-g)}+\frac{C \gamma^{4 / 3}}{(\xi-g)^{5 / 3}}
$$

Consequently, using the maximality of $\left(x_{1}, t_{1}\right)$ and recalling that $\xi-g \geq \eta>0$,

$$
\sup _{B\left(x_{0}, R\right) \times\left[t_{0}-T, t_{0}\right]} w^{2} \leq \frac{C \mu^{2}}{(\xi-g)^{2}}+\left.\frac{C \gamma^{4 / 3}}{(\xi-g)^{8 / 3}}\right|_{(x, t)=\left(x_{1}, t_{1}\right)} \leq C \mu^{2}+C \gamma^{4 / 3} .
$$

This proves (3.37) in this case. Thus, we can now assume that (3.39) is satisfied. Then, recalling (2.6), (2.8) and (2.12), we see that, in $Q_{R, T}$,

$$
\begin{gathered}
w \leq w\left(x_{1}, t_{0}-T\right)=\frac{\left(F^{\prime}(u)\right)^{2}|\nabla v|^{2}}{\xi-G(u)^{2}}\left(x_{1}, t_{0}-T\right) \\
=\frac{\left(F^{\prime}(u)\right)^{2}|\nabla u|^{2}}{u^{2}(\xi-G(u))^{2}}\left(x_{1}, t_{0}-T\right) \leq \tau_{u}^{2},
\end{gathered}
$$

which establishes (3.37) in this case.

We now suppose that (3.40) is satisfied. In such a case, we have that, in $Q_{R, T}$,

$$
w \leq w\left(x_{1}, t_{1}\right)=\frac{\left(F^{\prime}(u)\right)^{2}|\nabla u|^{2}}{u^{2}(\xi-G(u))^{2}}\left(x_{1}, t_{1}\right) \leq \sigma_{u}^{2},
$$

whence the proof of (3.37) is complete.

Lemma 3.7. In the setting of Theorem 1.5, if $x \in B\left(x_{0}, R-\rho\right)$ and $t \in\left[t_{0}-T+\delta, t_{0}\right]$,

$$
w(x, t) \leq C\left(\mu+\gamma^{2 / 3}+\frac{1}{\rho(R-\rho)}+\frac{\sqrt{k_{+}}}{\rho}+\frac{1}{\delta}+\frac{1}{\rho^{2}}\right),
$$

for some $C>0$, depending only on $\eta, a_{0}, \kappa$ and $\Gamma$. Here, $\mu$ and $\gamma$ are the quantities defined in (1.21) and (1.24), respectively. 
Proof. Let $\theta \in(0,1)$ to be conveniently chosen in what follows. Let also $\psi$ be as in (3.13) and $\phi$ be as in Lemma 3.2. We define $\Phi(x, t):=\psi(x) \phi(t)$ and $\widetilde{w}:=w \Phi$. Suppose that the maximum of $\widetilde{w}$ in $Q_{R, T}$ is reached at some point $\left(x_{1}, t_{1}\right)$. Since $\Phi$ vanishes along the parabolic boundary, we know that $x_{1} \in B\left(x_{0}, R\right)$ and $t_{1} \in$ $\left(t_{0}-T, t_{0}\right]$. As a consequence,

$$
\Delta \widetilde{w}\left(x_{1}, t_{1}\right) \leq 0, \quad \nabla \widetilde{w}\left(x_{1}, t_{1}\right)=0 \quad \text { and } \quad \widetilde{w}_{t}\left(x_{1}, t_{1}\right) \geq 0 .
$$

Combining this information with (3.5), we obtain that, at the point $\left(x_{1}, t_{1}\right)$,

$$
\begin{aligned}
0 \geq & \frac{a_{0} \kappa(\xi-g) w^{2} \Phi}{4}-a \lambda w\langle\nabla \Phi, \nabla g\rangle-\frac{C \mu^{2} \Phi}{(\xi-g)}-\frac{C \gamma^{4 / 3} \Phi}{(\xi-g)^{5 / 3}} \\
& -\frac{a g^{\prime} w|\nabla \Phi|^{2}}{\Phi}+\left.\frac{\left(a g^{\prime} \Delta \Phi-\Phi_{t}\right) w}{2}\right|_{(x, t)=\left(x_{1}, t_{1}\right)}
\end{aligned}
$$

From (3.23), (3.24) and (3.43), we deduce that

$$
0 \geq \frac{a_{0} \kappa(\xi-g) w^{2} \Phi}{16}-\frac{C \mu^{2} \Phi}{(\xi-g)}-\frac{C \gamma^{4 / 3} \Phi}{(\xi-g)^{5 / 3}}-\frac{C \Phi^{4 \theta-3}(\xi-g)}{\rho^{4}}+\left.\frac{\left(a g^{\prime} \Delta \Phi-\Phi_{t}\right) w}{2}\right|_{(x, t)=\left(x_{1}, t_{1}\right)} .
$$

Now, from (3.28),

$$
\begin{aligned}
& \frac{-a g^{\prime} w \Delta \Phi}{2}=\frac{-a g^{\prime} w \phi \Delta \psi}{2} \\
& \quad \leq \frac{a_{0} \kappa(\xi-g) w^{2} \psi \phi}{32}+\frac{C \psi^{2 \theta-1} \phi(\xi-g)}{\rho^{2}(R-\rho)^{2}}+\frac{C k_{+} \psi^{2 \theta-1} \phi(\xi-g)}{\rho^{2}}+\frac{C \psi^{2 \theta-1} \phi(\xi-g)}{\rho^{4}},
\end{aligned}
$$

and from (3.36),

$$
\frac{w \Phi_{t}}{2}=\frac{w \psi \phi_{t}}{2} \leq \frac{a_{0} \kappa(\xi-g) w^{2} \phi}{64}+\frac{C \phi^{\theta} \psi}{\delta^{2}(\xi-g)}
$$

From (3.44), (3.45) and (3.46) we obtain that, at the point $\left(x_{1}, t_{1}\right)$,

$$
\begin{aligned}
\frac{a_{0} \kappa(\xi-g) w^{2} \Phi}{64} \leq & \frac{C \mu^{2} \Phi}{(\xi-g)}+\frac{C \gamma^{4 / 3} \Phi}{(\xi-g)^{5 / 3}}+\frac{C \Phi^{4 \theta-3}(\xi-g)}{\rho^{4}}+\frac{C \psi^{2 \theta-1} \phi(\xi-g)}{\rho^{2}(R-\rho)^{2}} \\
& +\frac{C k_{+} \psi^{2 \theta-1} \phi(\xi-g)}{\rho^{2}}+\frac{C \psi^{2 \theta-1} \phi(\xi-g)}{\rho^{4}}+\frac{C \phi^{\theta} \psi}{\delta^{2}(\xi-g)} .
\end{aligned}
$$

We see that $0 \leq \Phi \leq 1$, and that $\Phi=1$ for every $x \in B\left(x_{0}, R-\rho\right)$ and $t \in\left[t_{0}-T+\delta, t_{0}\right]$. Thus, if $x \in B\left(x_{0}, R-\rho\right)$ and $t \in\left[t_{0}-T+\delta, t_{0}\right]$, choosing $\theta:=3 / 4$,

$$
\begin{aligned}
& w^{2}(x, t)=w^{2}(x, t) \Phi^{2}(x, t)=\widetilde{w}^{2}(x, t) \leq \widetilde{w}^{2}\left(x_{1}, t_{1}\right)=w^{2}\left(x_{1}, t_{1}\right) \Phi^{2}\left(x_{1}, t_{1}\right) \\
& \quad \leq \frac{C \mu^{2}}{(\xi-g)^{2}}+\frac{C \gamma^{4 / 3}}{(\xi-g)^{8 / 3}}+\frac{C}{\rho^{4}}+\frac{C}{\rho^{2}(R-\rho)^{2}}+\frac{C k_{+}}{\rho^{2}}+\left.\frac{C}{\delta^{2}(\xi-g)^{2}}\right|_{(x, t)=\left(x_{1}, t_{1}\right)},
\end{aligned}
$$

that, recalling (1.15), yields the desired estimate in (3.42). 


\section{Completion of the proof of Theorem 1.5}

In this section, we provide the proof of Theorem 1.5. To this end, we use the notation

$$
\begin{aligned}
& \widetilde{\mathcal{C}}:=\mu+\gamma^{2 / 3}, \\
\widetilde{\mathcal{T}} & :=\frac{1}{\delta} \\
\text { and } \quad \widetilde{\mathcal{S}} & :=\frac{1}{\rho^{2}}+\frac{1}{\rho(R-\rho)}+\frac{\sqrt{k_{+}}}{\rho} .
\end{aligned}
$$

With this notation, gathering together the estimates in Lemmata 3.4, 3.5, 3.6 and 3.7, we obtain the following statement.

Corollary 4.1. In the setting of Theorem 1.5, the function $w$ can be estimated by

$$
\begin{aligned}
C \widetilde{\mathcal{E}}+\left(\tau_{u}^{2}+C \widetilde{\mathcal{S}}\right) & \text { in } B\left(x_{0}, R-\rho\right) \times\left[t_{0}-T, t_{0}\right], \\
C \widetilde{\mathcal{E}}+\left(\sigma_{u}^{2}+C \widetilde{\mathcal{T}}\right) & \text { in } B\left(x_{0}, R\right) \times\left[t_{0}-T+\delta, t_{0}\right], \\
C \widetilde{\mathcal{E}}+\left(\sigma_{u}^{2}+\tau_{u}^{2}\right) & \text { in } B\left(x_{0}, R\right) \times\left[t_{0}-T, t_{0}\right], \\
C \widetilde{\mathcal{E}}+C(\widetilde{\mathcal{S}}+\widetilde{\mathcal{T}}) & \text { in } B\left(x_{0}, R-\rho\right) \times\left[t_{0}-T+\delta, t_{0}\right],
\end{aligned}
$$

for some $C>0$.

Hence, considering the more convenient term in any common domain, we deduce from Corollary 4.1 that:

Corollary 4.2. In the setting of Theorem 1.5, at any point in $Q_{R, T}$, we have that

$$
\begin{aligned}
w \leq C \widetilde{\mathcal{C}}+ & {\left[\min \left\{\sigma_{u}^{2}+\tau_{u}^{2}, \sigma_{u}^{2}+C \widetilde{\mathcal{T}}, \tau_{u}^{2}+C \widetilde{\mathcal{S}}, C(\widetilde{\mathcal{T}}+\widetilde{\mathcal{S}})\right\} \chi_{B\left(x_{0}, R-\rho\right) \times\left[t_{0}-T+\delta, t_{0}\right]}\right.} \\
& +\left(\sigma_{u}^{2}+\min \left\{\tau_{u}^{2}, C \widetilde{\mathcal{T}}\right\}\right) \chi_{\left(B\left(x_{0}, R\right) \backslash B\left(x_{0}, R-\rho\right)\right) \times\left[t_{0}-T+\delta, t_{0}\right]} \\
& +\left(\tau_{u}^{2}+\min \left\{\sigma_{u}^{2}, C \widetilde{\mathcal{S}}\right\}\right) \chi_{B\left(x_{0}, R-\rho\right) \times\left[t_{0}-T, t_{0}-T+\delta\right]} \\
& \left.+\left(\sigma_{u}^{2}+\tau_{u}^{2}\right) \chi_{\left(B\left(x_{0}, R\right) \backslash B\left(x_{0}, R-\rho\right)\right) \times\left[t_{0}-T, t_{0}-T+\delta\right]}\right],
\end{aligned}
$$

for some $C>0$.

Completion of the proof of Theorem 1.5. Recalling (3.18), we write that

$$
w=\frac{\left(F^{\prime}(u)\right)^{2}|\nabla u|^{2}}{u^{2}(\xi-G(u))^{2}} .
$$

This and (4.2) give that

$$
\begin{aligned}
\left.\frac{\left(F^{\prime}(u)\right)^{2} \mid}{u^{2}\left(\left.\xi u\right|^{2}\right.} \leq C(u)\right)^{2} & \leq C \widetilde{\mathcal{C}}+\left[\min \left\{\sigma_{u}^{2}+\tau_{u}^{2}, \sigma_{u}^{2}+C \widetilde{\mathcal{T}}, \tau_{u}^{2}+C \widetilde{\mathcal{S}}, C(\widetilde{\mathcal{T}}+\widetilde{\mathcal{S}})\right\} \chi_{B\left(x_{0}, R-\rho\right) \times\left[t_{0}-T+\delta, t_{0}\right]}\right. \\
& +\left(\sigma_{u}^{2}+\min \left\{\tau_{u}^{2}, C \widetilde{\mathcal{T}}\right\}\right) \chi_{\left(B\left(x_{0}, R\right) \backslash B\left(x_{0}, R-\rho\right)\right) \times\left[t_{0}-T+\delta, t_{0}\right]} \\
& +\left(\tau_{u}^{2}+\min \left\{\sigma_{u}^{2}, C \widetilde{\mathcal{S}}\right\}\right) \chi_{B\left(x_{0}, R-\rho\right) \times\left[t_{0}-T, t_{0}-T+\delta\right]} \\
& \left.+\left(\sigma_{u}^{2}+\tau_{u}^{2}\right) \chi_{\left(B\left(x_{0}, R\right) \backslash B\left(x_{0}, R-\rho\right)\right) \times\left[t_{0}-T, t_{0}-T+\delta\right]}\right] .
\end{aligned}
$$


Taking the square root and recalling (1.25), we obtain the desired result stated in Theorem 1.5.

\section{Proof of Corollary 1.6}

We now deduce a local estimate in $Q_{R / 2, T / 2}$ as a special case of the global one obtained in Theorem 1.5.

Proof of Corollary 1.6. By taking $\delta:=T / 2$ and $\rho:=R / 2$, we reduce the quantities $\mathcal{T}$ and $\mathcal{S}$ in (1.25) to

$$
\mathcal{T}=\sqrt{\frac{2}{T}} \quad \text { and } \quad \mathcal{S}=\frac{4}{R}+\frac{\sqrt{2} \sqrt[4]{k_{+}}}{\sqrt{R}}
$$

Consequently, we deduce from (1.28) that

$$
\iota \leq C(\mathcal{T}+\mathcal{S}) \leq C\left(\frac{1}{R}+\frac{1}{\sqrt{T}}+\frac{\sqrt[4]{k_{+}}}{\sqrt{R}}\right) .
$$

Furthermore, by (1.27), we know that $\mathcal{B}_{1}=\mathcal{B}_{2}=\mathcal{B}_{3}=0$ in $Q_{R / 2, T / 2}$ and therefore we deduce from Theorem 1.5 that, for each $(x, t) \in Q_{R / 2, T / 2}$,

$$
\begin{aligned}
& G^{\prime}(u(x, t))|\nabla u(x, t)| \leq(C \mathcal{C}+\iota \mathcal{J}(x, t))(\xi-G(u(x, t))) \\
& \leq C\left(\sqrt{\mu}+\sqrt[3]{\gamma}+\frac{1}{R}+\frac{1}{\sqrt{T}}+\frac{\sqrt[4]{k_{+}}}{\sqrt{R}}\right)(\xi-G(u(x, t))),
\end{aligned}
$$

as desired.

\section{Appendix A. A direct proof Showing that Corollary 1.6 implies Corollary 1.4 (i.e., Cor. 9 in [22])}

Goal of this appendix is to give a direct proof of Corollary 1.4 from the statement of Corollary 1.6. To this end, in the setting given by the statement of Corollary 1.4, we define $F(s):=s^{p}$. Let also

$$
\widetilde{T}:=M^{p-1} T \quad \text { and } \quad \widetilde{u}(x, t):=\frac{u\left(x, M^{1-p}\left(t-t_{0}\right)+t_{0}\right)}{M} .
$$

We observe that if $t \in\left[t_{0}-\widetilde{T}, t_{0}\right]$ then $M^{1-p}\left(t-t_{0}\right)+t_{0} \in\left[t_{0}-T, t_{0}\right]$. Consequently, if $(x, t) \in Q_{R, \widetilde{T}}$,

$$
\partial_{t} \widetilde{u}(x, t)=\frac{\partial_{t} u\left(x, M^{1-p}\left(t-t_{0}\right)+t_{0}\right)}{M^{p}}=\frac{\Delta u^{p}\left(x, M^{1-p}\left(t-t_{0}\right)+t_{0}\right)}{M^{p}}=\Delta \widetilde{u}^{p}(x, t) .
$$

Also, $0<\widetilde{u} \leq 1$, hence we can exploit Corollary 1.6, with $T$ replaced by $\widetilde{T}, u$ replaced by $\widetilde{u}$ and $M$ replaced by 1 . Moreover, in (1.14) we pick any $s_{0} \geq 2^{\frac{1}{1-p}}$ and we have that

$$
G(s)=p \int_{s_{0}}^{s} h^{p-2} d h=\frac{p}{1-p}\left(\frac{1}{s_{0}^{1-p}}-\frac{1}{s^{1-p}}\right) .
$$


Then, choosing $\eta:=\frac{p}{2(1-p)}$ and $\xi:=0$, we have that, for all $s \in(0,1]$,

$$
\xi-G(s)=\frac{p}{1-p}\left(\frac{1}{s^{1-p}}-\frac{1}{s_{0}^{1-p}}\right)
$$

and, as a byproduct,

$$
\xi-G(s) \geq \frac{p}{1-p}\left(1-\frac{1}{s_{0}^{1-p}}\right) \geq \frac{p}{2(1-p)}=\eta .
$$

Furthermore,

$$
\frac{\sqrt{n}\left|F^{\prime \prime}(s)\right| s}{F^{\prime}(s)}=\sqrt{n}(1-p)
$$

whence, setting $\kappa:=\sqrt{n}\left(p-1+\frac{1}{\sqrt{n}}\right)>0$,

$$
1-\frac{\sqrt{n}\left|F^{\prime \prime}(s)\right| s}{F^{\prime}(s)}=\kappa>0
$$

Moreover, for all $s \in(0,1]$,

$$
\begin{aligned}
& 2 F^{\prime}(s)-\frac{\sqrt{n}\left|F^{\prime \prime}(s)\right| s}{F^{\prime}(s)}(\xi-G(s))=2 p s^{p-1}-\sqrt{n}(1-p) \frac{p}{1-p}\left(\frac{1}{s^{1-p}}-\frac{1}{s_{0}^{1-p}}\right) \\
& =\frac{2 p}{s^{1-p}}-p \sqrt{n}\left(\frac{1}{s^{1-p}}-\frac{1}{s_{0}^{1-p}}\right)=\frac{p(2-\sqrt{n})}{s^{1-p}}+\frac{p \sqrt{n}}{s_{0}^{1-p}} \\
& \quad \geq p(2-\sqrt{4})+\frac{p \sqrt{n}}{s_{0}^{1-p}} \geq \frac{p \sqrt{n}}{s_{0}^{1-p}} \geq 0
\end{aligned}
$$

From this, (A.3) and (A.4), we see that the conditions in (1.12), (1.15) and (1.17) are fulfilled.

We now check that (1.16) is also satisfied (and, from the technical point of view, this step is the one that makes assumption (1.16) more convenient than (1.3)). To this end, we remark that, in light of (A.2),

$$
\xi-G(s)=\frac{p s^{p-1}}{1-p}-\frac{p s_{0}^{p-1}}{1-p}
$$

and therefore, for every $s \in(0,1]$,

$$
\frac{F^{\prime}(s)}{\xi-G(s)}=\frac{(1-p) s^{p-1}}{s^{p-1}-s_{0}^{p-1}}=\frac{1-p}{1-\left(s / s_{0}\right)^{1-p}}
$$

and consequently

$$
\frac{F^{\prime}(s)}{\xi-G(s)} \leq \frac{1-p}{1-\left(1 / s_{0}\right)^{1-p}} \leq 2(1-p)
$$


and this shows that condition (1.16) is fulfilled here with $\Gamma:=2(1-p)$.

Therefore, we can utilize Corollary 1.6 and conclude that, if $(x, t) \in Q_{R / 2, \widetilde{T} / 2}$,

$$
\begin{aligned}
& \frac{G^{\prime}(\widetilde{u}(x, t))|\nabla \widetilde{u}(x, t)|}{\xi-G(\widetilde{u}(x, t))}=\frac{|\nabla G(\widetilde{u}(x, t))|}{\xi-G(\widetilde{u}(x, t))} \\
& \quad \leq C\left(\frac{1}{R}+\frac{1}{\sqrt{\widetilde{T}}}+\sqrt{k}\right)=C\left(\frac{1}{R}+\frac{M^{\frac{1-p}{2}}}{\sqrt{T}}+\sqrt{k}\right) .
\end{aligned}
$$

Thus, recalling (A.5), we find that

$$
\xi-G(s)=\frac{s G^{\prime}(s)}{1-p}-\frac{p s_{0}^{p-1}}{1-p}
$$

and then (A.8) gives that

$$
(1-p) \frac{G^{\prime}(\widetilde{u}(x, t))|\nabla \widetilde{u}(x, t)|}{\widetilde{u}(x, t) G^{\prime}(\widetilde{u}(x, t))-p s_{0}^{p-1}} \leq C\left(\frac{1}{R}+\frac{M^{\frac{1-p}{2}}}{\sqrt{T}}+\sqrt{k}\right) .
$$

We can now send $s_{0} \rightarrow+\infty$ and (up to renaming constants) conclude that, for every $(x, t) \in Q_{R / 2, \widetilde{T} / 2}$,

$$
\frac{|\nabla \widetilde{u}(x, t)|}{\widetilde{u}(x, t)} \leq C\left(\frac{1}{R}+\frac{M^{\frac{1-p}{2}}}{\sqrt{T}}+\sqrt{k}\right),
$$

which, scaling back the time variable, leads to the desired result in (1.7).

Remark A.1. We stress that in this paper we are not addressing the optimality of the range of powers $p$ taken into account in the porous medium equation dealt with in Corollary 1.4 (rather, the main goal of this appendix was to show how to obtain some results in the literature, such as Corollary 9 in [22], as a byproduct of our main results). In a sense, we do not expect the range of $p$ presented here to be optimal and it can be expected that broader intervals in $p$ could be addressed by combining our methods with those in [29] (see in particular Remark 1.1 in [29]).

\section{Appendix B. The CASE of Equation (1.31)}

To emphasize the possible role of the additional function $H$ in the evolution equation (1.8), we present here a specific application (without aiming at the greatest possible generality):

Corollary B.1. Let $\mathcal{M}$ be a complete Riemannian manifold of dimension $n$ with $\operatorname{Ric}(\mathcal{M}) \geq-k$, for some $k \geq 0$. Let $u=u(x, t)$ be a positive solution of (1.31) in $Q_{R, T}$, with $0<m \leq u \leq M$ for some $M>0, m>0, \varepsilon>0$, $a_{0} \in(0,1), q>0, p$ as in $(1.6)$ and $a \in C^{1}(\mathbb{R})$ such that $a(t) \in\left[a_{0}, a_{0}^{-1}\right]$ for all $t \in \mathbb{R}$.

Let also

$$
\mathcal{F}(R, T):=\sup _{(x, t) \in Q_{R, T}} \frac{|\nabla u(x, t)|}{u(x, t)} \quad \text { and } \quad \mathcal{H}(R, T):=\sup _{(x, t) \in Q_{R, T}} \frac{\left|D^{2} u(x, t)\right|}{(u(x, t))^{3-p-q}} .
$$

Then, there exists $C>0$, depending only on $n, a_{0}, q$, and $p$ such that

$$
\mathcal{F}\left(\frac{R}{2}, \frac{T}{2}\right) \leq C\left(\frac{\sqrt{k} M^{\frac{1-p}{2}}}{m^{\frac{1-p}{2}}}+\sqrt[3]{\varepsilon} M^{\frac{2-2 p}{3}}(\mathcal{F}(R, T))^{\frac{q-1}{3}} \sqrt[3]{\mathcal{H}(R, T)}+\frac{1}{R}+\frac{M^{\frac{1-p}{2}}}{\sqrt{T}}+\frac{\sqrt[4]{k_{+}}}{\sqrt{R}}\right) .
$$


Proof. We let $\widetilde{T}$ and $\widetilde{u}$ as in (A.1) and $\widetilde{a}(t):=a\left(M^{1-p}\left(t-t_{0}\right)+t_{0}\right)$. Let also

$$
F(s):=s^{p} \quad \text { and } \quad H(\omega):=\varepsilon M^{q-p}|\omega|^{q} .
$$

In this way, we have that $\frac{m}{M} \leq \widetilde{u} \leq 1$ and, by (1.31),

$$
\begin{aligned}
\partial_{t} \widetilde{u}(x, t) & =\frac{\partial_{t} u\left(x, M^{1-p}\left(t-t_{0}\right)+t_{0}\right)}{M^{p}} \\
& =\frac{a\left(M^{1-p}\left(t-t_{0}\right)+t_{0}\right) \Delta u^{p}\left(x, M^{1-p}\left(t-t_{0}\right)+t_{0}\right)+\varepsilon\left|\nabla u\left(x, M^{1-p}\left(t-t_{0}\right)+t_{0}\right)\right|^{q}}{M^{p}} \\
& =\widetilde{a}(t) \Delta \widetilde{u}^{p}(x, t)+\frac{\varepsilon|\nabla \widetilde{u}(x, t)|^{q}}{M^{p-q}} \\
& =\widetilde{a}(t) \Delta(F(\widetilde{u})+H(\nabla \widetilde{u}),
\end{aligned}
$$

and thus we see that (B.3) is a special case of (1.8).

As already remarked in Appendix A, we have that conditions (1.11), (1.12), (1.15), (1.16) and (1.17) are satisfied by $F$ (with suitable $s_{0}, \xi, \eta, \Gamma$ and $\kappa$ depending only on $n$ and $p$ ).

Moreover, we have that $\widetilde{a} \in\left[a_{0}, a_{0}^{-1}\right]$, whence we deduce from (1.18), (1.20) and (A.7) that

$$
\begin{aligned}
\mu & =\mu_{1} \\
\leq & \sup _{(x, t) \in Q_{R, \widetilde{T}}}\left(a_{0}^{-1} k p(\widetilde{u}(x, t))^{p-1}+\frac{\varepsilon(p-1) M^{q-p}|\nabla \widetilde{u}(x, t)|^{q}}{\widetilde{u}(x, t)}\right. \\
& \left.\quad-\frac{\varepsilon M^{q-p}|\nabla \widetilde{u}(x, t)|^{q}}{\widetilde{u}(x, t)}+\frac{2 \varepsilon(1-p) M^{q-p}|\nabla \widetilde{u}(x, t)|^{q}}{\widetilde{u}(x, t)}\right)_{+} \\
= & \sup _{(x, t) \in Q_{R, \widetilde{T}}}\left(a_{0}^{-1} k p(\widetilde{u}(x, t))^{p-1}-\frac{p \varepsilon M^{q-p}|\nabla \widetilde{u}(x, t)|^{q}}{\widetilde{u}(x, t)}\right)_{+} \\
\leq & \sup _{(x, t) \in Q_{R, \widetilde{T}}}\left(a_{0}^{-1} k p(\widetilde{u}(x, t))^{p-1}\right)_{+} \\
\leq & \frac{k p M^{1-p}}{a_{0} m^{1-p}} .
\end{aligned}
$$

Also, owing to (1.19), (1.22) and (1.23),

$$
\begin{aligned}
\gamma & =\gamma_{3} \\
& \leq \sup _{(x, t) \in Q_{R, \widetilde{T}}} p q \varepsilon M^{q-p}(\widetilde{u}(x, t))^{p-2}|\nabla \widetilde{u}(x, t)|^{q-1}\left|D^{2} \widetilde{u}(x, t)\right| \\
& =\sup _{(x, t) \in Q_{R, T}} p q \varepsilon M^{2-2 p}(u(x, t))^{p-2}|\nabla u(x, t)|^{q-1}\left|D^{2} u(x, t)\right| \\
& \leq p q \varepsilon M^{2-2 p}(\mathcal{F}(R, T))^{q-1} \mathcal{H}(R, T) .
\end{aligned}
$$


From (A.2) (and sending $s_{0} \rightarrow+\infty$ ), utilizing Corollary 1.6, and renaming $C$ line after line, for every $(x, t) \in$ $Q_{R / 2, T / 2}$,

$$
\begin{aligned}
\sup _{Q_{R / 2, T / 2}} \frac{|\nabla u|}{u} & =\sup _{Q_{R / 2, \widetilde{T} / 2}} \frac{|\nabla \widetilde{u}|}{\widetilde{u}} \\
& \leq C\left(\sqrt{\mu}+\sqrt[3]{\gamma}+\frac{1}{R}+\frac{1}{\sqrt{\widetilde{T}}}+\frac{\sqrt[4]{k_{+}}}{\sqrt{R}}\right) \\
& \leq C\left(\frac{\sqrt{k} M^{\frac{1-p}{2}}}{\sqrt{a_{0}} m^{\frac{1-p}{2}}}+\sqrt[3]{\varepsilon} M^{\frac{2-2 p}{3}}(\mathcal{F}(R, T))^{\frac{q-1}{3}} \sqrt[3]{\mathcal{H}(R, T)}+\frac{1}{R}+\frac{M^{\frac{1-p}{2}}}{\sqrt{T}}+\frac{\sqrt[4]{k_{+}}}{\sqrt{R}}\right),
\end{aligned}
$$

that gives the desired result.

We remark that estimate (B.2) is certainly nonstandard in bounding $\mathcal{F}\left(\frac{R}{2}, \frac{T}{2}\right)$ with the obviously larger term $\mathcal{F}(R, T)$ and with the term $\mathcal{H}(R, T)$ that involves higher derivatives: nevertheless, estimate (B.2) is nontrivial, since these larger or higher order objects occur with a lower exponent when $q<4$ and are modulated by the (possibly small) structural parameter $\varepsilon$.

In this spirit, we point out a quantitative result on the oscillations of ancient solutions which easily follows from Corollary B.1:

Corollary B.2. Assume that

$\mathcal{M}$ is a complete Riemannian manifold of dimension $n$ with nonnegative Ricci curvature.

Let $u$ be a positive, bounded and smooth solution of the evolution equation

$$
\partial_{t} u=a(t) \Delta u^{p}+\varepsilon|\nabla u|^{q}
$$

in $\mathcal{M} \times(-\infty, 0]$, for some $a \in C^{1}((-\infty, 0])$ which is positive, bounded and bounded away from zero, some $\varepsilon>0$, $p \in\left(1-\frac{1}{\sqrt{n}}, 1\right]$ and $q \in(0,4)$.

Assume that

$$
\mathcal{F}_{\star}:=\sup _{\mathcal{M} \times(-\infty, 0]} \frac{|\nabla u|}{u}<+\infty \quad \text { and } \quad \mathcal{H}_{\star}:=\sup _{\mathcal{M} \times(-\infty, 0]} \frac{\left|D^{2} u\right|}{u^{3-p-q}}<+\infty .
$$

Then, there exists $C>0$ depending only on $n, a, q$ and $p$ such that

$$
\mathcal{F}_{\star} \leq C \varepsilon^{\frac{1}{4-q}} M^{\frac{2-2 p}{4-q}} \mathcal{H}_{\star}^{\frac{1}{4-q}}
$$

where $M$ is the supremum of $u$.

Proof. We let $\mathcal{F}(R, T)$ and $\mathcal{H}(R, T)$ be as in (B.1). With this notation, and recalling (B.4), we can exploit (B.2) with $t_{0}:=0$ and any given $x_{0} \in \mathcal{M}$, thereby finding that, for every $R>0, T>0, x \in B\left(x_{0}, R / 2\right)$ and $t \in$ $[-T / 2,0]$,

$$
\mathcal{F}\left(\frac{R}{2}, \frac{T}{2}\right) \leq C\left(\sqrt[3]{\varepsilon} M^{\frac{2-2 p}{3}}(\mathcal{F}(R, T))^{\frac{q-1}{3}} \sqrt[3]{\mathcal{H}(R, T)}+\frac{1}{R}+\frac{M^{\frac{1-p}{2}}}{\sqrt{T}}\right)
$$


Consequently, we can send $R \rightarrow+\infty$ and $T \rightarrow+\infty$ and obtain that

$$
\mathcal{F}_{\star} \leq C \sqrt[3]{\varepsilon} M^{\frac{2-2 p}{3}} \mathcal{F}_{\star}^{\frac{q-1}{3}} \sqrt[3]{\mathcal{H}_{\star}},
$$

from which the desired result follows.

\section{Appendix C. A Liouville-type Result}

In this appendix we point out that suitable classification results for ancient solutions under appropriate pointwise bounds follows directly from uniform interior estimates. We do not address the most general statement here, but just provide the following one as a simple example:

Corollary C.1. Assume that

$\mathcal{M}$ is a complete Riemannian manifold of dimension $n$ with nonnegative Ricci curvature.

Let $u$ be a positive and smooth solution of the evolution equation

$$
u_{t}=a(t) \Delta(F(u))
$$

in $\mathcal{M} \times(-\infty, 0]$, for some $a \in C^{1}((-\infty, 0])$ which is positive, bounded and bounded away from zero, and for some $F \in C^{2}(0,+\infty)$ satisfying (1.11), (1.12), (1.15), (1.16) and (1.17).

Assume that

$$
\sup _{\substack{x \in B_{R} \\ t \in[-T, 0]}} u(x, t)=o(R)+o(\sqrt{T})
$$

near infinity. Then, $u$ is constant.

Proof. We exploit Corollary 1.6. For this, we observe that we can take $k:=0$, owing to (C.1). Furthermore, comparing (C.2) with (1.18), (1.19), (1.20), (1.21), (1.22), (1.23) and (1.24), we see that $\mu=\gamma=0$.

For this reason, for every $R>0, T>0, x \in B_{R / 2}$ and $t \in[-T / 2,0]$ the use of Corollary 1.6 leads to

$$
G^{\prime}(u(x, t))|\nabla u(x, t)| \leq C\left(\frac{1}{R}+\frac{1}{\sqrt{T}}\right)(\xi-G(u(x, t))),
$$

for a suitable structural constant $C$.

This and (1.14) give that

$$
|\nabla u(x, t)| \leq C\left(\frac{1}{R}+\frac{1}{\sqrt{T}}\right) \frac{(\xi-G(u(x, t))) u(x, t)}{F^{\prime}(u(x, t))} .
$$

Hence, in light of (1.17),

$$
|\nabla u(x, t)| \leq C\left(\frac{1}{R}+\frac{1}{\sqrt{T}}\right) \frac{F^{\prime}(u(x, t))}{\left|F^{\prime \prime}(u(x, t))\right|},
$$


and thus, by (1.12),

$$
|\nabla u(x, t)| \leq C\left(\frac{1}{R}+\frac{1}{\sqrt{T}}\right) u(x, t)
$$

up to renaming $C$ line after line.

Therefore, utilizing (C.3), for every $x \in B_{R / 2}$ and $t \in\left[-R^{2} / 2,0\right]$,

$$
|\nabla u(x, t)| \leq \frac{C}{R} \sup _{B_{R} \times\left[-R^{2}, 0\right]} u=\frac{o(R)}{R} .
$$

Sending now $R \rightarrow+\infty$ we obtain the desired result.

With respect to (C.3), we do not address here the problem of the optimal growth at infinity needed to obtain nontrivial solutions. As a matter of fact, we do not expect condition (C.3) to be optimal (indeed, at least when $a$ is independent of $t$ and $F$ is a suitable power, milder growth assumptions lead to suitable classification results, see e.g. Theorems 1.3 and 1.5 in [29]).

Acknowledgements. Serena Dipierro and Enrico Valdinoci are members of INdAM and AustMS. Serena Dipierro has been supported by the Australian Research Council DECRA DE180100957 "PDEs, free boundaries and applications". Enrico Valdinoci has been supported by the Australian Laureate Fellowship FL190100081 "Minimal surfaces, free boundaries and partial differential equations".

\section{REFERENCES}

[1] D.G. Aronson and P. Benilan, Regularite des solutions de l'equation des milieuxporeuxdans $\mathrm{R}^{N}$. C. R. Acad. Sci. Paris Ser. $A-B 288$ (1979) A103-A105.

[2] Giles Auchmuty and David Bao, Harnack-type inequalities for evolution equations. Proc. Am. Math. Soc. 122 (1994) $117-129$.

[3] J. Baptiste Joseph Fourier, Theorie analytique de la chaleur, Cambridge Library Collection, Cambridge University Press, Cambridge (2009).

[4] S. Benachour and P. Laurencot, Global solutions to viscous Hamilton-Jacobi equations with irregular initial data. Commun. Partial Differ. Equ. 24 (1999) 1999-2021.

[5] D. Bianchi and A.G. Setti, Laplacian cut-offs, porous and fast diffusion on manifolds and other applications. Calc. Var. Partial Differ. Equ. 57 (2018) 33.

[6] M. Bonforte, G. Grillo and J.L. Vazquez, Fast diffusion flow on manifolds of nonpositive curvature. J. Evol. Equ. 8 (2008) 99-128.

[7] D. Castorina and C. Mantegazza, Ancient solutions of semilinear heat equations on Riemannianmanifolds. Atti Accad. Naz. Lincei Rend. Lincei Mat. Appl. 28 (2017) 85-101.

[8] D. Castorina and C. Mantegazza, Ancient solutions of superlinear heat equations on Riemannian manifolds. Commun. Contemp. Math. 23 (2021) 2050033.

[9] C. Cavaterra, S. Dipierro, A. Farina, Z. Gao and E. Valdinoci, Pointwise gradient bounds for entire solutions of elliptic equations with non-standard growth conditions and general nonlinearities. J. Differ. Equ. 270 (2021) 435-475.

[10] C. Cavaterra, S. Dipierro, Z. Gao and E. Valdinoci, Global gradient estimates for a general type of nonlinear parabolic equations. Preprint arXiv arXiv:2006.00263 (2020).

[11] R. Dal Passo and S. Luckhaus, A degenerate diffusion problem not in divergence form. J. Differ. Equ. 69 (1987) 1-14.

[12] A. Friedman, Partial differential equations of parabolic type. Prentice-Hall, Inc., Englewood Cliffs, NJ (1964).

[13] G. Grillo and M. Muratori, Smoothing effects for the porous medium equation on Cartan-Hadamard manifolds. Nonlinear Anal. 131 (2016) 346-362.

[14] G. Grillo, M. Muratori and J.L. Vazquez, The porous medium equation on Riemannianmanifolds with negative curvature. The large-time behavior. Adv. Math. 314 (2017) 328-377.

[15] R.S. Hamilton, A matrix Harnack estimate for the heat equation. Commun. Anal. Geom. 1 (1993) $113-126$.

[16] M.A. Herrero and M. Pierre, The Cauchy problem for $u_{t}=\Delta u^{m}$ when $0<m<1$. Trans. Am. Math. Soc. 291 (1985) $145-158$.

[17] E.P. Hsu, Estimates of derivatives of the heat kernel on a compact Riemannian manifold. Proc. Am. Math. Soc. 127 (1999) 3739-3744.

[18] P. Li and S.-T. Yau, On the parabolic kernel of the Schrodinger operator. Acta Math. 156 (1986) 153-201.

[19] P. Lu, L. Ni, J.-L. Vazquez and C. Villani, Local Aronson-Benilan estimates and entropy formulae for porous medium and fast diffusion equations on manifolds. J. Math. Pures Appl. 91 (2009) 1-19. 
[20] L. Ma, Gradient estimates for a simple elliptic equation on complete non-compact Riemannian manifolds. J. Funct. Anal. 241 (2006) 374-382.

[21] L. Ma and Y. An, The maximum principle and the Yamabeflow, Partial differential equations and their applications (Wuhan, 1999). World Sci. Publ., River Edge, NJ (1999) 211-224.

[22] L. Ma, L. Zhao and X. Song, Gradient estimate for the degenerate parabolic equation $u_{t}=\Delta F(u)+H(u)$ on manifolds. $J$. Differ. Equ. 244 (2008) 1157-1177.

[23] P. Malliavin and D.W. Stroock, Short time behavior of the heat kernel and its logarithmic derivatives. J. Differ. Geom. 44 (1996) 550-570.

[24] P. Souplet and Q.S. Zhang, Sharp gradient estimate and Yau's Liouville theorem for the heat equation on noncompact manifolds. Bull. London Math. Soc. 38 (2006) 1045-1053.

[25] D.W. Stroock and J. Turetsky, Upper bounds on derivatives of the logarithm of the heat kernel. Commun. Anal. Geom. 6 (1998) 669-685.

[26] M. Ughi, A degenerate parabolic equation modelling the spread of an epidemic. Ann. Mat. Pura Appl. 143 (1986) 385-400.

[27] J. Vazquez, The porous medium equation, Oxford Mathematical Monographs. The Clarendon Press, Oxford University Press, Oxford (2007).

[28] W. Wang, Harnack differential inequalities for the parabolic equation $u_{t}=\mathcal{L} F(u)$ on Riemannian manifolds and applications. Acta Math. Sin. (Engl. Ser.) 33 (2017) 620-634.

[29] X. Xu, Gradient estimates for $u_{t}=\Delta F(u)$ on manifolds and some Liouville-type theorems. J. Differ. Equ. 252 (2012) $1403-1420$.

[30] Y. Yang, Gradient estimates for a nonlinear parabolic equation on Riemannian manifolds. Proc. Am. Math. Soc. 136 (2008) 4095-4102.

[31] X. Zhu, Hamilton's gradient estimates and Liouville theorems for fast diffusion equations on noncompact Riemannian manifolds. Proc. Am. Math. Soc. 139 (2011) 1637-1644.

[32] X. Zhu, Hamilton's gradient estimates and Liouville theorems for porous medium equations on noncompact Riemannian manifolds. J. Math. Anal. Appl. 402 (2013) 201-206. 